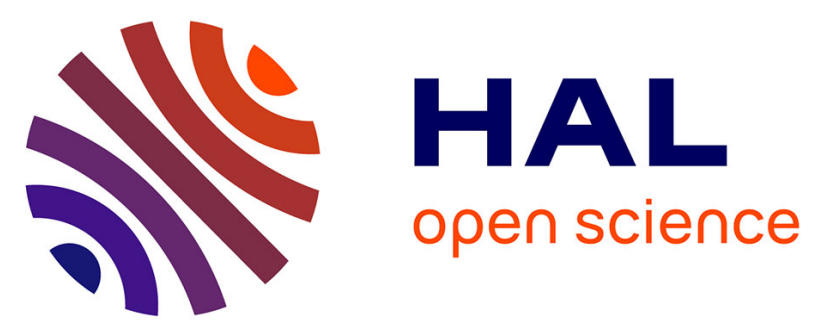

\title{
Coronin 1C promotes triple-negative breast cancer invasiveness through regulation of MT1-MMP traffic and invadopodia function
}

Alessia Castagnino, Antonio Castro-Castro, Marie Irondelle, Alan Guichard, Catalina Lodillinsky, Laetitia Fuhrmann, Sophie Vacher, Sonia Agüera-González, Anna Zagryazhskaya-Masson, Maryse Romao, et al.

\section{To cite this version:}

Alessia Castagnino, Antonio Castro-Castro, Marie Irondelle, Alan Guichard, Catalina Lodillinsky, et al. Coronin $1 \mathrm{C}$ promotes triple-negative breast cancer invasiveness through regulation of MT1-MMP traffic and invadopodia function. Oncogene, 2018, 37 (50), pp.6425-6441. 10.1038/s41388-018-0422x . hal-02377262

\section{HAL Id: hal-02377262 https://hal.science/hal-02377262}

Submitted on 23 Nov 2019

HAL is a multi-disciplinary open access archive for the deposit and dissemination of scientific research documents, whether they are published or not. The documents may come from teaching and research institutions in France or abroad, or from public or private research centers.
L'archive ouverte pluridisciplinaire HAL, est destinée au dépôt et à la diffusion de documents scientifiques de niveau recherche, publiés ou non, émanant des établissements d'enseignement et de recherche français ou étrangers, des laboratoires publics ou privés. 
1 Coronin 1C promotes triple-negative breast cancer invasiveness through 2 regulation of MT1-MMP traffic and invadopodia function

3 Alessia Castagnino ${ }^{1}$, Antonio Castro-Castro ${ }^{1}$, Marie Irondelle ${ }^{1,2}$, Alan Guichard ${ }^{1}$,

4 Catalina Lodillinsky ${ }^{1,3,4}$, Laetitia Fuhrmann ${ }^{5}$, Sophie Vacher ${ }^{6}$, Sonia Agüera-

5 González ${ }^{1}$, Anna Zagryazhskaya-Masson ${ }^{1}$, Maryse Romao ${ }^{7}$, Carole El Kesrouani ${ }^{5}$,

6 Angelika A. Noegel ${ }^{8}$, Thierry Dubois ${ }^{9}$, Graça Raposo ${ }^{7}$, James E. Bear ${ }^{10}$, Christoph

7 S. Clemen ${ }^{11,8}$, Anne Vincent-Salomon ${ }^{5}$, Ivan Bièche ${ }^{6,12}$ and Philippe Chavrier ${ }^{1, *}$

81 Institut Curie, PSL Research University, CNRS UMR144, Membrane and 9 Cytoskeleton Dynamics group, 26 rue d'Ulm, F-75005, Paris, France

102 Institut Curie, PSL Research University, Cell and Tissue Imaging Facility (PICT11 IBiSA), 26 rue d'UIm, F-75005, Paris, France

$12{ }^{3}$ Universidad de Buenos Aires. Facultad de Medicina. Instituto de Oncología A. H. 13 Roffo. Área de Investigación. San Martin 5481, Buenos Aires C1417DTB, Argentina

$14{ }^{4}$ Member of Consejo Nacional de Investigaciones Científicas y Técnicas (CONICET), 15 Argentina

165 Institut Curie, PSL Research University, Pathology-Genetics-Immunology 17 Department, 26 rue d'UIm, F-75005, Paris, France

186 Institut Curie, PSL Research University, Pharmacogenomic Unit, Department of 19 Genetics, 26 rue d'Ulm, F-75005, Paris, France

$20 \quad{ }^{7}$ Institut Curie, PSL Research University, CNRS UMR144, Biogenesis and Functions 21 of Lysosome-Related Organelles group, 26 rue d'Ulm, F-75005, Paris, France 
$22{ }^{8}$ Center for Biochemistry, Institute of Biochemistry I, Medical Faculty, University of

23 Cologne, Joseph-Stelzmann-Str. 52, 50931 Cologne, Germany

$24{ }^{9}$ Institut Curie, PSL Research University, Translational Research Department, Breast

25 Cancer Biology Group, Paris, France

2610 UNC Lineberger Comprehensive Cancer Center and the Department of Cell

27 Biology and Physiology, The University of North Carolina at Chapel Hill, Chapel Hill, 28 NC 27599, USA

2911 Department of Neurology, Heimer Institute for Muscle Research, University 30 Hospital Bergmannsheil, Ruhr-University Bochum, Bürkle-de-la-Camp-Platz 1, 44789 31 Bochum, Germany

32 EA7331, Paris Descartes University, Sorbonne Paris Cité, Faculty of 33 Pharmaceutical and Biological Sciences, 4 avenue de l'observatoire, F-75006, Paris, 34 France

Conflict of interest disclosure statement: Authors have no conflict of interest to declare.

Running title: Coronin $1 \mathrm{C}$ regulates breast cancer invasion

Keywords: Coronin 1C, MT1-MMP traffic, invadopodia, breast cancer, invasion

corresponding author: Philippe Chavrier, Institut Curie, CNRS UMR144, 26 rue

42 d'Ulm, F-75005, Paris, France. Phone: +33156246359, e-mail:

43 philippe.chavrier@curie.fr 


\section{Abstract}

45 Membrane type 1-matrix metalloproteinase (MT1-MMP), a membrane-tethered 46 protease, is key for matrix breakdown during cancer invasion and metastasis. 47 Assembly of branched actin networks by the Arp2/3 complex is required for MT1MMP traffic and formation of matrix-degradative invadopodia. Contrasting with the well-established role of actin filament branching factor cortactin in invadopodia

50 function during cancer cell invasion, the contribution of coronin-family debranching 51 factors to invadopodia-based matrix remodeling is not known. Here, we investigated 52 the contribution of coronin $1 \mathrm{C}$ to the invasive potential of breast cancer cells. We 53 report that expression of coronin $1 \mathrm{C}$ is elevated in invasive human breast cancers, 54 correlates positively with MT1-MMP expression in relation with increased metastatic 55 risk and is a new independent prognostic factor in breast cancer. We provide 56 evidence that, akin to cortactin, coronin $1 \mathrm{C}$ is required for invadopodia formation and 57 matrix degradation by breast cancer cells lines and for 3D collagen invasion by 58 multicellular spheroids. Using intravital imaging of orthotopic human breast tumor 59 xenografts, we find that coronin $1 \mathrm{C}$ accumulates in structures forming in association 60 with collagen fibrils in the tumor microenvironment. Moreover, we establish the role of coronin $1 \mathrm{C}$ in the regulation of positioning and trafficking of MT1-MMP-positive 62 endolysosomes. These results identify coronin $1 \mathrm{C}$ as a novel player of the multi63 faceted mechanism responsible for invadopodia formation, MT1-MMP surface 64 exposure and invasiveness in breast cancer cells. 


\section{Introduction}

Tumor cell escape and dissemination to distant sites are hallmarks of metastasis, the leading cause of cancer-related death. During breast cancer progression, a key step is the transition from ductal carcinoma in situ (DCIS) - a mass of proliferative cells inside the mammary duct surrounded by an intact myoepithelium and by the basement membrane (BM) - to invasive breast cancers. DCIS-to-invasive breast cancer transition is associated with breakdown of the myoepithelium and the underlying BM and increased metastasis risk. The capacity of cancer cells to remodel extracellular matrix (ECM) barriers is essential for cancer progression and metastasis 1. Proteolytic remodeling of ECM components by cancer cells mobilizes a group of transmembrane matrix metalloproteinases (MMPs), which includes membrane type 1 (MT1)-MMP (aka MMP14) ${ }^{1}$. We recently reported that MT1-MMP is up-regulated in invasive hormone receptor- and epidermal growth factor receptor 2 (HER2)-negative triple-negative breast cancers (TNBCs) as compared to DCIS lesions and normal breast tissue and overexpression predicts the invasive potential of cancerous lesions 2 .

Experimental data based on cancer cell models revealed that ECM remodeling by carcinomatous cells is focused to the pericellular zone ${ }^{3,4}$. Matrix gaps and tunnels forming overtime support infiltrating cell passage through the BM and through the fibrous collagen microenvironment ${ }^{3}$. ECM breakdown is mediated by specialized cellular structures of metastatic cells called invadopodia, which form at the plasma membrane in association with ECM fibrils and require actin assembly for their formation ${ }^{5}$. Invadopodia are the sites of surface exposure and accumulation of MT1MMP ${ }^{6-8}$. Due to difficulties inherent to the submicrometric size and transient nature of invadopodia, fewer studies addressed their formation, dynamics and structure in 
vivo. However, recent work based on emerging intravital microscopy technics, reported the existence of invadopodia-like protrusions in relation with matrix remodeling and metastasis in the natural tumor microenvironment ${ }^{9,10}$.

Polymerization of invadopodial actin is a multistep process that requires activation of the Arp2/3 complex by nucleation promoting factor (NPF) Wiskott-Aldrich syndrome like protein (N-WASP) ${ }^{11}$. Regulators of branched actin network dynamics including cortactin (CTTN) and cofilin are likewise essential for invadopodia formation ${ }^{6,12}$. The actin-binding protein CTTN promotes actin network assembly by facilitating N-WASP displacement from Arp2/3 complex and stabilizing Arp2/3 complex at branches ${ }^{13,14}$. Moreover, CTTN promotes branch stabilization by antagonizing members of the WD40 domain-containing F-actin binding coronin family members, which control debranching and Arp2/3-nucleated filament network disassembly ${ }^{15-17}$. Additionally, coronins bind to and inhibit nucleation by the Arp2/3 complex ${ }^{15,18,19}$. All together, these data point to some coordinated functions of CTTN and coronin in the regulation of branched actin network assembly and dynamics.

In carcinoma cells, altered phosphorylation and up-regulation of CTTN are associated with increased invadopodia density, promoting invasion and tumor aggressiveness ${ }^{12,}{ }^{20-24}$. Similarly, the coronin-family protein coronin $1 \mathrm{C}$ (CORO1C aka coronin-3, CRN2) is up-regulated in human cancers including gastric cancers, hepatocellular carcinomas and brain tumors and is correlated with increased invasiveness and metastasis ${ }^{24-28}$. In addition, experimental data have shown that CORO1C is required for degradation of gelatin as a matrix mimic and for formation of invasive protrusions by human glioblastoma cells ${ }^{28,29}$. 
114 late endosome (LE)/lysosome compartments in returning internalized MT1-MMP to

115 invadopodia forming at plasma membrane/ECM contact sites ${ }^{7,8,23,30-32}$. Docking of

116 MT1-MMP-positive LE/lysosomes to the invadopodial plasma membrane allows

117 surface exposure of the protease possibly through the formation of tubular

118 connections between the limiting membrane of LE/lysosomes and the plasma

119 membrane ${ }^{8,32,33}$. The pathological relevance of this recycling circuitry has been

120 recently established in the context of the invasion program of TNBCs $23,31,32$. A

121 characteristic feature of this mechanism is the presence of F-actin/CTTN-enriched

122 puncta on MT1-MMP-positive LE/lysosomes, which depend on the Arp2/3 complex

123 and its activator WASH complex for their formation ${ }^{8,23,34,35}$. Perturbation of WASH

124 function affects endolysosomal F-actin/CTTN puncta, interferes with the trafficking of

125 MT1-MMP vesicles to the invadopodial plasma membrane and inhibits MT1-MMP

126 surface exposure and invasion ${ }^{8,}{ }^{32}$. Interestingly, a study in macrophages has

127 provided evidence for a role of coronin $1 \mathrm{~A}(\mathrm{CORO} 1 \mathrm{~A})$ in controlling endolysosomal

128 actin assembly in the context of cholesterol trafficking and clearance ${ }^{36}$. In addition,

129 coordinated functions of CTTN and coronin 1B (CORO1B aka coronin-2) have been

130 implicated in trafficking and plasma membrane docking of multivesicular LEs and

131 regulation of exosome secretion from these compartments in conjunction with the

132 small GTPase Rab27a function 37, 38. All together, these data suggest some

133 cooperative functions of CTTN and coronins in the regulation of endolysosomal actin

134 dynamics and trafficking and exocytosis of LE/lysosomal cargo proteins including 135 MT1-MMP.

136 Here we show that CORO1C is up-regulated in invasive breast cancers and 137 correlates positively with MT1-MMP expression in TNBCs in relation with poor 138 prognosis. Additionally, our results indicate that CORO1C plays a dual function in 
139 MT1-MMP-dependent pericellular matrix degradation by regulating invadopodia 140 formation and by controlling the positioning of MT1-MMP storage endolysosomes. 


\section{Results}

142 CORO1C is associated with poor outcome in breast cancer. Expression of

143 CORO1A, -B and -C type I coronin family members and pro-invasive MT1-MMP was 144 analyzed by immunoblotting in a panel of 30 breast cancer cell lines. These cell lines 145 were stratified in Normal-like, Luminal, TNBC and HER2 ${ }^{+}$subtypes based on the expression patterns of estrogen and progesterone receptors (ER and PR) and HER2 amplification ${ }^{39}$. Expression of hematopoietic-specific CORO1A was detected in Jurkat leukemic T-cells while levels were low to barely detectable in the breast cancer cell lines (Fig. 1AB). In contrast, CORO1B expression was detected in most

150 breast cancer cell lines irrespective of subtypes (Fig. 1A and C). Expression of

151 CORO1C was mostly restricted to TNBC cells similar to MT1-MMP expression 152 pattern (Fig. 1A and DE). Expression of CORO1B and CORO1C mRNAs was 153 analyzed by quantitative RT-PCR in invasive breast cancers using a retrospective 154 cohort of 446 patients with long-term follow-up. It allowed us to examine if variations 155 in CORO1B and $-C$ mRNA expression had a value for patient prognosis (Table S3). 156 We found that $12.1 \%$ of breast tumors overexpressed CORO1B mRNA ( $>3$ relative to 157 normal tissues, not shown). Highest CORO1B expression levels were observed in 158 HER2 tumors and positively correlated with Ki-67 proliferation marker (Table S4). 159 However, we did not see an impact of CORO1B expression on the metastasis-free survival (MFS) of breast cancer patients (not shown).

161 Tumors expressing high levels of CORO1C showed more often ER and PR 162 negativity, correlated with high Ki-67 and epidermal growth factor receptor (EGFR) 163 levels and were more frequently of an advanced grade indicative of an association 164 with bad prognosis (Table S5). Most importantly, CORO1C up-regulation was 165 associated with significantly shorter MFS (Fig. 1F). Multivariate analysis using a Cox 
166 proportional hazard model revealed independent predictive value for MFS of lymph

167 node status, tumor size and grade and CORO1C mRNA expression parameters,

168 indicating that CORO1C expression is a new independent prognostic factor in breast

169 cancer (Table S6, $P=0.0029)$.

170 Based on available RT-qPCR data in the same cohort ${ }^{2}$, we found a strong positive 171 correlation of CORO1C and MT1-MMP mRNAs (Fig. 1G). Correlation of CORO1C 172 and MT1-MMP expression was similarly observed at the protein level by 173 immunoblotting analysis of a subset of tumor samples selected based on low and 174 high mRNA levels; while there was no obvious correlation with CORO1B or CTTN 175 expression in these tumor samples (Fig. S1AB). Importantly, we analyzed the 176 association of CORO1C and MT1-MMP transcript levels with clinical outcome and 177 found significantly increased metastatic risk in patients with both high MT1-MMP and 178 CORO1C mRNA levels (Fig. 1H). The prognostic significance of 'MT1-MMP and 179 CORO1C expression level' persisted in Cox multivariate regression analysis (Table $180 \quad \mathrm{~S} 7, \mathrm{P}=0.00007)$.

181 Next, we focused our study on CORO1C showing strongest correlation with pro182 invasive MT1-MMP and previously implicated in cancer progression and metastasis 25, 26, 28. Changes in CORO1C protein levels in epithelial cancer cells were 184 investigated by immunohistochemistry (IHC) analysis of a tissue microarray (TMA) of 185 invasive breast cancers and adjacent peritumoral tissues from an independent cohort 186 of 136 patients (Table S8). Specificity of CORO1C IHC staining was established by analysis of human MCF10DCIS.com breast cancer-derived cells knocked down for 188 CORO1C expression (Fig. S1C). In peritumoral epithelial tissues, we detected low 189 cytoplasmic levels of CORO1C in luminal cells and strong expression in 190 myoepithelial cells (Fig. 2AB). CORO1C staining was observed in the cytoplasm of 
191 breast carcinoma cells (Fig. 2AB). Quantification and analysis of CORO1C H-score

192 (intensity multiplied by percentage of positively stained cells) revealed significantly

193 higher levels of CORO1C in invasive cancer relative to normal breast tissues (Fig.

194 2C), in agreement with RNA expression data (Table S5) and protein analysis in

195 breast cancer cell lines (Fig. 1A and D). CORO1C levels were highest in TNBC

196 subtype although difference with other subtypes was not statistically significant (Fig.

197 2D). Therefore we concluded that CORO1C expression was up-regulated in invasive

198 breast cancers at the mRNA and protein levels, particularly in TNBCs and correlated

199 with worse prognosis and increased metastasis risk in association with MT1-MMP

200 up-regulation. At the mechanistic level, these data suggested some involvement of

201 CORO1C in MT1-MMP-dependent pericellular ECM proteolysis activity during breast

202 tumor progression and dissemination.

203 CORO1C is required for invadopodia function and tumor cell invasion. ${ }^{\text {GFP }}$ CORO1C was stably overexpressed in MDA-MB-231 cells used as a TNBC model

(Fig. S2A). These cells invaded through the 3D fibrous type I collagen network with an elongated morphology typical of this highly invasive mesenchymal cell line (Fig. 3A, low magnification inset and Supplementary Movie S1). CTTN and ${ }^{\text {GFP }}$ CORO1C co-localized in lamellipodia at the edge of invasive protrusions extending within the collagen gel (Fig. 3A, low magnification inset). In addition, arc-shape structures enriched for CTTN and CORO1C were visible in association with collagen fibrils in front of the nucleus, which was located at the rear of the cell (Fig. 3A, inset 1). Strong

212 pericellular collagenolysis was observed in association with the bulbous nuclear cell

213 region as revealed by staining for neoepitope of MMP-cleaved type I collagen (Fig.

$2143 \mathrm{~B}$, yellow). When cells were plated on top of a 2D layer of collagen fibrils, CORO1C 215 was similarly found at the cell edge as well as in curvilinear structures forming in 
association with the underlying collagen fibrils, which were positive for the

217 invadopodia protein TKS5 (Fig. 3C) ${ }^{8,40}$. Collectively, our observations indicated that

218 CORO1C accumulated at proteolytically active invadopodia forming in association

219 with confining collagen fibrils.

220 The effect of ${ }^{\text {GFP }}$ CORO1C overexpression on the invasive capacity of MDA-MB-231

221 cells in 3D collagen was assessed. Multicellular spheroids of MDA-MB-231 cells

222 overexpressing GFP or ${ }^{\text {GFP }}$ CORO1C were formed and embedded in the type I

223 collagen gel and invasion was monitored after two days. Overexpression of

$224{ }^{\text {GFP }}$ CORO1C significantly increased the invasive potential of MDA-MB-231 cells in

225 the 3D collagen gel (Fig. 3DE). CORO1C-dependent invasion required MMP activity

226 as shown by inhibition in the presence of generic GM6001 MMP inhibitor (Fig. 3E).

227 The proinvasive potential of CORO1C was generalized using multicellular spheroids

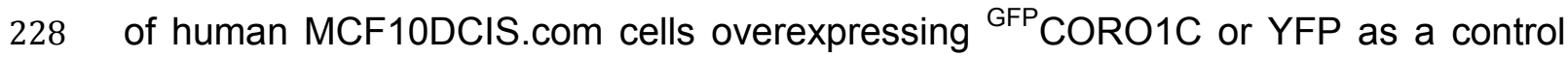

229 (Fig. S3AB).

230 Actin polymerization is essential for invadopodia formation and function and

231 CORO1C is known to regulate branched actin dynamics. We therefore investigated

232 the contribution of CORO1C to the formation of TKS5-positive invadopodia in MDA-

233 MB-231 cells plated on top of a collagen fibril layer as in Fig. 3C. Overexpression of

234 CORO1C increased significantly the formation of TKS5-positive invadopodia (Fig.

235 3F). ${ }^{\text {GFP }}$ CORO1C ${ }_{\mathrm{R} 28 \mathrm{D} / 2 \mathrm{KE}}$ harboring the R28D, K418E, K419E, K427E, and K428E

236 mutations, which is defective for F-actin binding ${ }^{41}$, was diffusely distributed indicating

237 that CORO1C association to invadopodia required binding to F-actin similar to

238 CORO1B and -C recruitment to the lamellipodia ${ }^{41,42}$ (Fig. S3C). Remarkably, actin-

239 binding deficient $\mathrm{CORO}_{\mathrm{R} 28 \mathrm{D} / 2 \mathrm{KE}}$ exerted a dominant inhibitory effect on

240 invadopodia formation (Fig. 3F). An equivalent mutation in CORO1B has been shown 
241 to affect F-actin binding but no other molecular interactions ${ }^{15,42}$, which may explain

242 the observed dominant inhibitory potential by titration of important functional partners.

243 In addition, CORO1C was knocked-down by treatment with two independent siRNAs

244 leading to $>95 \%$ silencing of the protein with no effect on MT1-MMP or TKS5 levels

245 (Fig. S2C). Loss of CORO1C expression reduced by 50-60\% the capacity of MDA-

246 MB-231 cells to form TKS5-positive invadopodia in association with the collagen

247 fibrils (Fig. 3G and Fig. S3DE). CORO1B knockdown similarly decreased the

248 formation of TKS5-positive invadopodia by 50\% (Fig. 3G and Fig. S2D). Loss of

249 both CORO1B and -1C did not further reduce invadopodia formation, suggesting that

250 the two isoforms may function together as recently reported ${ }^{17}$ (Fig. 3G). Along this

251 line, we found that overexpressed $\mathrm{CORO}^{\mathrm{GFP}}$ accumulated together with MT1-

$252 \mathrm{MMP}^{\mathrm{mCh}}$ in invadopodia forming in association with collagen fibers (Fig. $3 \mathrm{H}$, inset 1

253 and Fig. S2B).

254 Consequences of modulation of CORO1C expression on pericellular matrix 255 degradation in 3D collagen were analyzed by staining of cleaved collagen. Collagen 256 proteolysis mediated by MT1-MMP was strongly up-regulated upon CORO1C 257 overexpression, while ECM degradation was inhibited by $\mathrm{CORO}_{\mathrm{R} 28 \mathrm{D} / 2 \mathrm{KE}}$ or 258 CORO1C silencing (Fig. 3I-K). Collectively, these data indicated that CORO1C had a 259 significant contribution to invadopodia formation and to the invasive program of 260 TNBC cells.

261 We found that CORO1C co-localized with CTTN at invadopodia (Fig. 3A). Using the 262 same assays, we observed that CTTN knockdown strongly decreased 3D collagen 263 invasion of MDA-MB-231 multicellular spheroids similar to the silencing of MT1-MMP 264 and CORO1C (Fig. S4A-C). In addition, knockdown of CTTN also correlated with a 265 strong reduction of pericellular collagen degradation (Fig. S4DE). Thus we concluded 
that up-regulation of CORO1C expression promoted the invasive potential of TNBC by favoring the formation of proteolytically active invadopodia and that CORO1B/1C and CTTN contributed to invadopodia activity suggesting some cooperative function.

COR01C puncta are observed in association with collagen fibers in breast

tumor cells in vivo. Our data identified CORO1C as a key invadopodia component of invasive MDA-MB-231 cells. In agreement with CORO1C requirement during collective invasion by epithelial MCF10DCIS.com breast cancer cells (Fig. S3AB), we also observed a strong association of ${ }^{\text {GFP }}$ CORO1C with TKS5-positive proteolytically active invadopodia forming in association with collagen fibrils in this cell line (Fig. S5A-C). Thus, the distribution of ${ }^{\mathrm{GFP}} \mathrm{CORO} 1 \mathrm{C}$, which localized to invadopodia in breast cancer cells was investigated in vivo. We used intravital imaging of mammary MDA-MB-231 and MCF10DCIS.com cell tumor xenografts to visualize ${ }^{\text {GFP }}$ CORO1C in relation with the tumor ECM microenvironment. MDA-MB-231/ ${ }^{\mathrm{GFP}} \mathrm{CORO} 1 \mathrm{C}$ tumor xenografts growing in the mammary gland of SCID mice upon fat pad injection were imaged using 2-photon laser scanning intravital microscopy combined with second harmonic generation (SHG) to visualize collagen fibrils (Fig. 4A). Accumulations of ${ }^{\mathrm{GFP}}$ CORO1C were visible in tumor cells adjacent to collagen fibers (Fig. 4A-C, arrows and insets 1-3). These structures were morphologically heterogeneous ranging from small (submicrometric) puncta (Fig. 4B and insets 1-2) to several $\mu \mathrm{m}$-long linear structures similar to curvilinear invadopodia observed in tumor cell lines in vitro (Fig.

4C and inset 3). Contrastingly, intravital imaging of fat pad tumors of MDA-MB-231 cells expressing ${ }^{\mathrm{GFP}} \mathrm{CORO} 1 \mathrm{C}_{\mathrm{R} 28 \mathrm{D} / 2 \mathrm{KE}}$ revealed a diffuse cytosolic distribution of the actin-binding defective CORO1C variant in tumor xenografts (Fig. 4D and inset 4). Therefore, similar to our observations in cultured cells, accumulation of CORO1C in association with collagen fibers in vivo required F-actin binding capacity of CORO1C. 
291 The distribution of CORO1C was similarly analyzed during the growth and invasion of

292 MCF10DCIS.com tumor xenograft generated using the intraductal (nipple-) injection

293 model ${ }^{43}$. We and others reported that injection of this cell line into the duct lumen in

294 SCID mice leads to the formation of DCIS tumor xenografts, which can further

295 progress into invasive lesions upon BM breaching depending on MT1-MMP activity ${ }^{2}$,

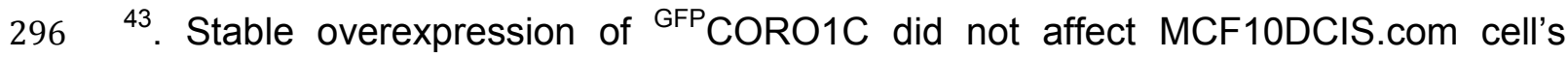

297 growth in culture (Fig. S5D). Whole-mount and tissue section staining of tumor

298 xenografts analyzed 4-5 weeks after intraductal injection revealed the presence of

299 large epithelial tumors growing within the mammary duct system of SCID mice

300 injected with control or GFPCORO1C-overexpressing MCF10DCIS.com cells (Fig.

301 S5E, F and H). Tumors presented a necrotic center and some invasive foci of

302 epithelial cells were visible within the mammary gland stroma (Fig. S5E, arrowhead).

303 Tumor burden (foci number and size) was increased in mammary glands injected

304 with ${ }^{\text {GFP}}$ CORO1C-overexpressing cells as compared to control MCF10DCIS.com

305 cells, although differences were not statistically significant 4 to 5-weeks after injection

306 (Fig. S5F-I). These data suggested that ${ }^{\text {GFP }}$ CORO1C-overexpression conferred some

307 growth advantage to MCF10DCIS.com epithelial tumors. Ductal GFPCORO1C-

308 expressing tumor xenografts were analyzed by intravital 2-photon imaging of GFP

309 and SHG signals. Intraductal tumors were detected as tumor mass surrounded by a

310 thick bed of collagen bundles tangential to the duct (Fig. 4EF). Some microinvasive

311 buds were visible consisting of few ${ }^{\text {GFP }}$ CORO1C-overexpressing epithelial cells that

312 migrated in collective manner within the type I collagen bed (Fig. 4EF and insets 5

313 and 6). Invasion of ${ }^{\text {GFP }}$ CORO1C cells progressed overtime in the collagen-enriched

314 stroma (compare inset 5 and 6 acquired at 1-week time interval). Intravital imaging at

315 later time point (7-weeks post injection) revealed frankly invasive tumor lesions (Fig. 
316 4G-I). The presence of ${ }^{\mathrm{GFP}} \mathrm{CORO1C}$ accumulations forming at the surface of tumor

317 MCF10DCIS.com cells in contact with intratumoral collagen fibers was visible similar

318 to MDA-MB-231 tumors (Fig. 4G-I and inset 7). Collectively, these data indicated that

319 CORO1C accumulated in structures reminiscent of ECM degradative invadopodia,

320 which formed in association with collagen fibrils in the invasive breast tumor

321 xenografts in vivo.

322 Loss of CORO1C causes MT1-MMP-positive LE/lysosome mispositioning and

323 collapse. We have shown that MT1-MMP trafficking to invadopodia depends on

324 actin dynamics on endolysosomes ${ }^{8,23}$. The distribution of ${ }^{\text {GFP }}$ CORO1C was analyzed

325 by live cell imaging by confocal microscopy in MDA-MB-231 cells expressing MT1-

$326 \mathrm{MMP}^{\mathrm{mCh}}$. As previously described, MT1-MMP ${ }^{\mathrm{mCh}}$ accumulated in vesicles that we

327 previously identified as LE/lysosomes ${ }^{7,32}$. In addition to ${ }^{\text {GFP }}$ CORO1C localization to

328 lamellipodia, we observed the accumulation of ${ }^{\text {GFP }}$ CORO1C in dynamic and discrete

329 puncta on a majority of MT1-MMP ${ }^{\mathrm{mCh}}$-positive vesicles (Fig. 5A and Supplementary

330 Movie S2). In contrast, F-actin-binding-deficient ${ }^{\text {GFP }}$ CORO1C $\mathrm{C}_{\mathrm{R} 28 \mathrm{D} / 2 \mathrm{KE}}$ was diffuse and

331 cytosolic (Fig. 5B). Endosomal CORO1C-enriched puncta were also positive for

332 CTTN, although a slight shift was visible between the localization of the two proteins

333 in line with the distinct roles played by these proteins in the actin branching cycle ${ }^{15,17}$

334 (Fig. 5C, inset). Similarly, CORO1B ${ }^{\text {GFP }}$-positive puncta were visible on MT1-MMP

335 LE/lysosomes (Fig. 3H, inset 2). Cryoimmunoelectron microscopy confirmed the

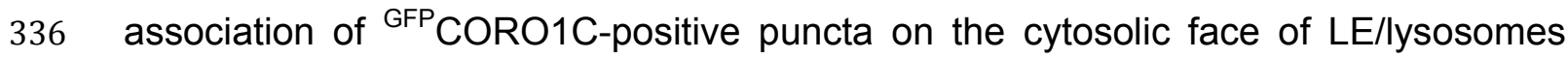

337 (Fig. 5D). All together, these data revealed that CORO1C and CORO1B localized to

338 endosomal actin-, CTTN-rich puncta, which depend on WASH and Arp2/3 complexes

339 for their formation and are implicated in MT1-MMP delivery to invadopodia in breast

340 cancer cells $^{8,23,32,34,35}$. 
341 We went on analyzing the consequences of CORO1C knockdown on the distribution

342 and morphology of MT1-MMP-positive LE/lysosomes. In control RNAi treated cells, 343 MT1-MMP-positive LE/lysosomes were distributed throughout the cell, with a 344 concentration in the central region of the cell (Fig. 5E and IJ). In cells silenced for 345 CORO1C, MT1-MMP-positive LE/lysosomes were also predominantly localized to 346 the cell center (Fig. 5F and I). In addition, we observed that reduced expression of 347 CORO1C resulted in a tight clustering of MT1-MMP-positive LE/lysosomes in $\sim 70 \%$ 348 of the cells (Fig. 5J). We recently identified the scaffolding proteins JIP4 and JIP3 as 349 key players in MT1-MMP endosome positioning by linking LE/lysosomes to the 350 microtubule motors kinesin-1 and dynein/dynactin ${ }^{32}$. As previously described ${ }^{32}$, 351 depletion of JIP3 and JIP4 resulted in MT1-MMP endosome dispersion toward the 352 cell periphery (Fig. 5G and IJ and Fig. S6A). Remarkably, triple knockdown of 353 JIP3/JIP4 and CORO1C partially restored some level of scattering of MT1-MMP354 positive endosomes (Fig. $5 \mathrm{H}$ and $\mathrm{IJ}$ ). All together, these data suggested that tight 355 clustering of MT1-MMP-positive LE/lysosomes induced upon loss of CORO1C 356 function required retrograde endosomal trafficking in a JIP3/JIP4-dependent manner.

357 Cells expressing MT1-MMP ${ }^{\mathrm{mCh}}$ were fixed and stained for JIP4 and CTTN, and

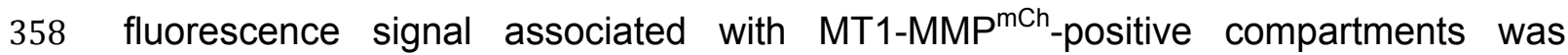
359 quantified. As previously reported ${ }^{32}$, JIP4, which is predominantly cytosolic, was 360 detected throughout the cytoplasm and in close proximity to CTTN-positive puncta on 361 the cytosolic face of MT1-MMP endosomes (Fig. 6A and quantification in panels DE, 362 see also Fig. S6C-C" for lower magnification images). Quantification of fluorescence 363 signal associated with clustered, enlarged MT1-MMP ${ }^{\mathrm{mCh}}$-positive endosomal 364 compartments in CORO1C-depleted cells revealed opposite effects on CTTN and 365 JIP4 association, i.e. CTTN association was diminished, while JIP4 accumulated on 
366 MT1-MMP compartments as compared to siNT-treated cells (Fig. 6B and DE and

367 Fig. S6D-E'). Additionally, CTTN knockdown did not alter significantly the association

368 of JIP4 with endosomal membranes (Fig. 6C and DE and Fig. S6B and FF').

369 We noticed that morphological changes observed in CORO1C-depleted cells 370 including enlargement and perinuclear clustering of LE/Lysosomal compartments

371 were reminiscent of cellular alterations typical of Niemann-Pick disease type C 372 (NPC). Indeed, cholesterol-enriched LEs cluster in the perinuclear area, as a consequence of defective LE/Lysosome traffic and cholesterol clearance in cells of

374 Iysosomal storage disease patients including NPC cells ${ }^{44}$. After fixation, MDA-MB-

375231 cells treated with control siRNA were stained with the fluorescent cholesterol-

376 binding probe filipin and scattered cholesterol-positive LE structures were observed

377 (Fig. S6G). Treatment of the cells with U18666A, a widely-used amphipathic steroid,

378 which blocks exit of cholesterol from LE/lysosomes and recapitulates the NPC

379 phenotype ${ }^{45}$, induced tight clustering of cholesterol-laden LE/Lysosomes in the 380 perinuclear cell region (Fig. S6H). Similarly, LE/Lysosome clusters forming in 381 CORO1C-depleted cells were strongly enriched for cholesterol indicative of defective 382 cholesterol exit from the clustered endosomes (Fig. S6IJ).

383 In order to get further insight at phenotypic alterations of MT1-MMP-positive 384 compartments induced by loss of CORO1C function, MDA-MB-231 cells knocked 385 down for CORO1C were analyzed by transmission electron microscopy. Figure $6 \mathrm{~F}$ 386 shows the distribution of LE/lysosomes in control cells. CORO1C depletion caused 387 various morphological alterations of LE/lysosomal compartments, which were 388 enlarged, aggregated and fused together and accumulated osmiophilic materials 389 including lamellar inclusions reminiscent of the NPC phenotype (Fig. 6G-I). 390 Collectively, these data indicated that CORO1C played a key role in MT1-MMP 
391 LE/lysosome positioning. Loss of CORO1C expression altered the association of 392 JIP4 (and CTTN) on endosomal membranes resulting in LE/lysosome clumps in the 393 perinuclear area possibly responsible for deficient recycling and surface-exposure of 394 MT1-MMP. 


\section{Discussion}

396 Previous findings indicated that CORO1C, a member of the coronin family of highly conserved F-actin-binding proteins and regulators of branched actin networks, contributes to invasiveness and metastasis in several cancer types including glioblastoma, primary effusion lymphoma and TNBC $24,26-29,46$. In addition, CORO1C

400 is a biomarker for invasive progression of hepatocellular carcinoma ${ }^{25}$. However, our 401 understanding of the cellular and molecular mechanisms underlying the role of 402 CORO1C in tumor invasion has remained limited.

403 Here, we analyzed the expression profile of CORO1C in breast cancer in relation 404 with MT1-MMP, the protease responsible for pericellular matrix degradation by carcinoma cells with major implications in the invasive potential and progression of breast tumors $2,47,48$. In a panel of cell lines representative of the breast cancer 407 molecular subtypes ${ }^{39}$, CORO1C protein expression was detected mainly in TNBC 408 cells similar to MT1-MMP ${ }^{2}$. CORO1B was broadly expressed irrespective of the 409 molecular subtypes, while expression of hematopoietic-specific CORO1A was low to 410 undetectable $^{16}$. Based on RT-qPCR analysis of a retrospective cohort of invasive 411 breast cancers, we found that CORO1C transcript was up-regulated breast cancers 412 and was associated with hormone receptor-negativity, high grade and metastasis 413 risk. In addition, co-up-regulation of CORO1C and MT1-MMP also correlated with 414 significantly higher metastases risk. 'High CORO1C expression' was an independent 415 prognostic factor of breast cancer in multivariate analysis and prognostic significance 416 of 'MT1-MMP and CORO1C expression level' persisted in Cox multivariate 417 regression analysis. Likewise, IHC analysis of invasive breast cancer TMAs 418 confirmed the up-regulation of CORO1C in breast carcinoma at the protein level as 419 compared to normal epithelial tissue. CORO1B mRNA was also up-regulated in a 
420 breast tumor subset, although with no impact on metastasis-free survival. Similar to

421 our findings in breast cancer, published data have correlated CORO1C expression

422 with high-grade gliomas and liver cancers and poor prognosis in stomach cancers ${ }^{25}$ 42327,28 , while no such correlation has been reported between CORO1B and cancer 424 progression. In conclusion, our data, which extend a previous analysis of publicly 425 accessible databases ${ }^{46}$, indicate that CORO1C is up-regulated in hormone receptor426 negative breast tumors and associates with poor outcome.

427 Several studies reported that coronins, including CORO1C, regulate lamellipodial 428 branched actin network and CORO1C activity impinges on cell migration and 429 invasion by cancer cells including MDA-MB-231 TNBC cells in vitro 28, 29, 46, 49. 430 Moreover, CORO1C was associated with invasive cell protrusions and has been 431 implicated in the degradation of the matrix mimic gelatin by human U373 432 glioblastoma-derived cells ${ }^{28,29}$. In the present study, we observed that CORO1C co433 localized with CTTN and TKS5 in matrix-degradative actin-based invadopodia 434 forming in association with type I collagen fibrils in TNBC cells. Importantly, 435 knockdown of CORO1C inhibited invadopodia formation, pericellular collagenolysis 436 and invasive migration in 3D collagen in two TNBC cell models with distinct 437 mesenchymal (MDA-MB-231) or epithelial (MCF10DCIS.com) features. Thus, our 438 work indicates that CORO1C is required both for single-cell and collective invasion 439 patterns. Association of CORO1B with invadopodia was also observed and CORO1B 440 was required for invadopodia formation. Moreover, loss of both CORO1C and 1B did 441 not further inhibited invadopodia formation, suggesting that both isoforms may 442 function as a complex as previously shown ${ }^{17}$.

443 Actin polymerization is a key component of invadopodia-based invasion program by 444 driving invasive cell protrusions through the matrix and maintaining tight apposition of 
445 surface-exposed MT1-MMP with confining ECM fibrils ${ }^{4,8}$. In addition, binding of 446 MT1-MMP cytosolic tail to the invadopodial actin network is thought to anchor MT1447 MMP to these structures ${ }^{50}$. Assembly of invadopodial actin requires activation of the 448 Arp2/3 complex by N-WASP ${ }^{8,11,50}$. An antagonism of CTTN and coronin functions 449 has been proposed in the control of a cycle of Arp2/3 branch stabilization and 450 destabilization ( ${ }^{15-17,51}$ and references herein). In addition, coronin and CTTN have 451 been implicated in the regulation of cofilin activity in actin filament turnover, which is 452 crucial during invadopodia assembly ${ }^{11,51}$. We observed that CORO1B/1C and CTTN 453 co-localized at invadopodia. Alike CORO1B/1C, CTTN has been identified as a key 454 regulator of invadopodia formation and its overexpression in various cancer types is 455 associated with tumor progression and metastasis ${ }^{6,12}$. Here, we found that 456 unbalanced CTTN and CORO1B/1C activity upon individual silencing (or inhibition) of 457 either proteins impairs invadopodia formation and function, emphasizing complex 458 and cooperative functions of CTTN and CORO1B/1C in the branching/debranching 459 cycle to replenish Arp2/3 complex and actin pools to promote invadopodia actin 460 dynamics and cancer cell invasion. Overall, our data suggest that CORO1B and 461 CORO1C function together in the mechanism of invadopodia formation and in matrix 462 remodeling. Yet, the reason for the specific association of CORO1C up-regulation 463 and not CORO1B - with cancer aggressiveness is unclear, we cannot rule out some 464 additional function of CORO1C related to its association with cancer aggressiveness.

465 Invadopodia's role in matrix degradation has been experimentally established, 466 however the existence and physiological relevance of invadopodia in cancer is 467 questionable because of a lack of direct evidence in vivo. Yet, recent studies 468 provided convincing evidence that invadopodia formation is essential for extravasation and intravasation of tumor cells and promotes lung metastasis in mice 
473 cancer cell intra- or extravasation in vivo ${ }^{9,10}$. Importantly, intravital imaging of

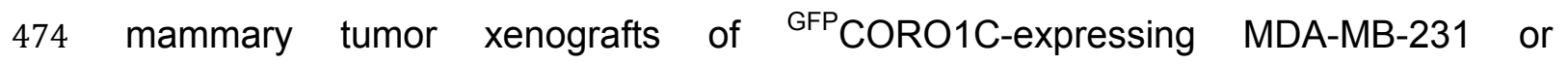
475

9, 10, 52 . Reports based on intravital imaging of tumor cells expressing fluorescentlytagged markers documented the existence of cytoplasmic protrusions identified as invadopodia based on CTTN, N-WASP or TKS5 enrichment and that correlated with MCF10DCIS.com cells revealed the presence of ${ }^{\text {GFP }}$ CORO1C accumulations adjacent to collagen fibers in the tumor microenvironment. However, evidence that these structures are proteolytically active is lacking and we cannot formally conclude that ${ }^{\text {GFP }}$ CORO1C accumulations represent bona fide invadopodia in vivo. Noticeably, ${ }^{\text {GFP }}$ CORO1C-positive structures were observed both in mesenchymal MDA-MB-231 cells and in strands of invasive epithelial MCF10DCIS.com cells supporting the implication of CORO1C in mesenchymal and collective invasion programs.

Branched actin networks also exist on intracellular compartments including endolysosomes where their main function is to sculpt and stabilize endosomal membrane microdomains involved in cargo sorting and recycling $34,35,53$. Endosomal actin patches are nucleated by the Arp2/3-complex upon WASH-mediated activation 34, 35 . Earlier on, we found that the WASH complex localizes to MT1-MMP-containing LE/lysosomes and its function is required for MT1-MMP trafficking to invadopodia ${ }^{8}$. In addition, several groups reported that depletion of WASH led to enlargement and massive tubulation of the endolysosomal system supporting the idea that WASHdependent actin assembly may regulate the fission of endosomal recycling tubules ${ }^{34}$, 35, 54 . CTTN was also found to associate with and regulate endosomal actin puncta on MT1-MMP-positive LE/Lysosomes, and it was reported that CTTN loss or deregulation led to the accumulation of enlarged LE/Lysosomes and defective endosomal traffic ${ }^{8,23,37,55}$. The present study provides corroborating evidence that in 
495 the absence of CORO1C, endolysosomal compartments dramatically enlarged and 496 collapsed in the perinuclear cell area. It has been argued that deficient cargo exit 497 exemplified by massive cholesterol accumulation and organelle obstruction may lead 498 to endosome enlargement ${ }^{54}$. Alteration of the endosomal actin coat may induce 499 endosomal collapse possibly through uncontrolled fusion of individual endosomes. 500 This assumption is supported by findings that interfering with endosomal actin 501 regulatory components such as WASH, CTTN, CORO1A or -C lead to perinuclear 502 redistribution and collapse of endolysosomal compartments ${ }^{34-37,54}$ (this study).

503 Moreover, we previously found that the related JIP3 and JIP4 scaffolding proteins 504 regulate the recruitment of minus-end and plus-end microtubule motor complexes to 505 WASH-positive domains ${ }^{32}$. Loss of JIP3 and JIP4 proteins led to the accumulation of 506 MT1-MMP compartments at the cell periphery indicating unbalanced minus/plus-end 507 motor activities (at minus-end dynein expense) ${ }^{32}$. Here we found that loss of 508 CORO1C moderately increases JIP3/JIP4 recruitment on MT1-MMP-positive 509 endosomes although the underlying mechanism remains unknown. Interestingly, 510 enhanced recruitment of JIP4 to endolysosomal compartments was recently shown 511 to induce endolysome collapse into the cell center ${ }^{56}$. Excess JIP3/JIP4 on MT1-MMP 512 endosomes is expected to promote their perinuclear accumulation due to increased 513 dynein activity, possibly favoring endosome collision and collapse. In agreement with 514 this assumption, we found that formation of endosomal clusters upon loss of 515 CORO1C was abrogated when JIP3/JIP4 were silenced. Also to be considered, 516 coordinated functions of coronins and CTTN were also implicated in the regulation of 517 endolysosome docking with the plasma membrane and exocytosis ${ }^{37,38}$. All together, 518 our work highlights a molecular framework based on interplay of WASH, CTTN, 
519 CORO1C and JIP3/JIP4 function in the regulation of endosome positioning and 520 dynamics as an essential component of MT1-MMP-based metastatic program. 
522 Cell culture. MDA-MB-231 cells (ATCC HTB-26) were grown in L15 medium 523 supplemented with $15 \%$ fetal calf serum and $2 \mathrm{mM}$ glutamine at $37^{\circ} \mathrm{C}$ in $1 \% \mathrm{CO}_{2}$.

524 MCF10DCIS.com cell line was purchased from Asterand and maintained in DMEM-

525 F12 medium with 5\% horse serum. MDA-MB-231 and MCF10DCIS.com cell lines 526 were checked monthly for mycoplasma contamination using a real-time PCR method 527 (MYCOPLASMACHECK, GATC Biotech AG).

Stable and transient transfection and siRNA treatment. MDA-MB-231 cells stably expressing MT1-MMP ${ }^{\mathrm{mCh}}$ have been previously described ${ }^{57}$. Lentiviral constructs encoding ${ }^{\mathrm{GFP}} \mathrm{CORO} 1 \mathrm{C}$ or ${ }^{\mathrm{GFP}} \mathrm{CORO} 1 \mathrm{C}_{\mathrm{R} 28 \mathrm{D} / 2 \mathrm{KE}}{ }^{41}$ cloned in pLKO.1 vector have been described ${ }^{29}$. MDA-MB-231 cells expressing ${ }^{\mathrm{GFP}} \mathrm{CORO} 1 \mathrm{C},{ }^{\mathrm{GFP}} \mathrm{CORO} 1 \mathrm{C}_{\mathrm{R} 28 \mathrm{D} / 2 \mathrm{KE}}$, ${ }^{\text {GFP }}$ CORO1C/MT1-MMP ${ }^{m C h},{ }^{G F P}$ CORO1C ${ }_{\text {R28D/2KE }} / M T 1-M M P^{m C h}$ and MCF10DCIS.com

533 cells stably expressing ${ }^{\text {GFP }}$ Coro1C were generated by lentiviral transduction as 534 described ${ }^{58}$. pLL7.0-m1B-EGFP construct encoding CORO1B ${ }^{\text {GFP }}$ was provided by

535 J.E. Bear. For transient expression, MDA-MB-231 cells were transfected with plasmid 536 constructs using AMAXA nucleofection (Lonza). Cells were analyzed by live cell 537 imaging 24-48 hr after transfection. For knockdown, MDA-MB-231 cells were treated 538 with the indicated siRNA (50 nM, see Table S1) using Lullaby (OZ Biosciences) and 539 analyzed 72 hours after treatment.

540 Antibodies and reagents. Antibodies used for this study are listed in Table S2.

541 GM6001 (Millipore) was diluted in ethanol and used at a concentration of $40 \mu \mathrm{M}$.

542 Filipin III (stock in DMSO, $0.1 \mathrm{mg} / \mathrm{ml} \mu \mathrm{M}$ working concentration) and U18666A (stock 543 in $\mathrm{H}_{2} \mathrm{O}, 10 \mu \mathrm{M}$ working concentration) were purchased from Sigma. 
544 Indirect immunofluorescence microscopy. Samples were fixed with $4 \%$

545 paraformaldehyde, permeabilized with $0.1 \%$ Triton $\mathrm{X}-100$, and then incubated with

546 specific antibodies (see Table S2). For quantification of CTTN or JIP4 on MT1-

$547 \mathrm{MMP}^{\mathrm{mCh}}$-containing endosomes, cells were stained with specific antibodies and z-

548 dimension series of images (z-stacks) were acquired with $0.2 \mu \mathrm{m}$ interval with a wide-

549 field Eclipse 90i Upright Microscope (Nikon) by mean of a piezoelectric motor (Physik

550 Instrument), a 100x Plan Apo VC 1.4 oil immersion objective and a cooled interlined

551 charge-coupled device (CCD) camera (CoolSnap HQ2, Roper Scientific). Images

552 were deconvolved ${ }^{59}$, and detection of CTTN or JIP4 on MT1-MMP ${ }^{\mathrm{mCh}}$-containing

553 endosomes from the median plane of the z-stack was performed using a CellProfiler

554 pipeline as previously described ${ }^{23,60}$. Briefly, MT1-MMPmCh-containing endosomes

555 were identified by thresholding and intensity-based watershed; CTTN or JIP4 spots in

556 a 3-pixel wide neighborhood around each $M T 1-M M P^{m C h}$-positive vesicle were

557 identified using a Laplacian of Gaussian filter followed by a watershed on the

558 automatically thresholded image. Finally, the total area of CTTN or JIP4 spots was

559 normalized over the total of MT1-MMPmCh-positive vesicles.

560 Live cell spinning disk confocal microscopy. For live cell confocal spinning disk

561 microscopy, MDA-MB-231 cells stably expressing ${ }^{\text {GFP }}$ Coro1C and MT1-MMP ${ }^{\mathrm{mCh}}$ or

$562{ }^{\mathrm{GFP}} \mathrm{CORO} 1 \mathrm{C}_{\mathrm{R} 28 \mathrm{D} / 2 \mathrm{KE}}$ and MT1-MMPmCh were plated on glass-bottom dishes (MatTek

563 Corporation) coated with cross-linked gelatin and kept in a humidified atmosphere at

$56437^{\circ} \mathrm{C}$ in $1 \% \mathrm{CO} 2$. Time-series were acquired using a spinning disk microscope

565 (Roper Scientific) based on a CSU X1 head (Yokogawa) mounted on the lateral port

566 of an inverted microscope (TE-2000U-Nikon) steered by MetaMorph software and

567 equipped with a 60x 1.45 NA oil-immersion objective, a Piezzo Z stage (Mat-City- 
568 Lab), a dual output laser launch that included 491- and 561-nm 50-mW DPSS lasers

569 (Roper Scientific) and a CCD camera (CoolSNAP HQ2).

570 Analysis of endosome distribution and clustering. For the analysis of the

571 distribution of MT1-MMP ${ }^{m C h}$-positive endosomes, a MATLAB software has been

572 developed to determine the relative position of each endosome to the cell centroid as 573 previously described ${ }^{61}$.

574 Invadopodia formation assay. Coverslips were layered with $100 \mu \mathrm{l}$ of a $2.2 \mathrm{mg} / \mathrm{ml}$ 575 solution of acid-extracted type I rat tail collagen (Corning) mixed with AlexaFluor 647576 conjugated type I collagen ( $5 \%$ final). After gelling for $3 \mathrm{~min}$ at $37^{\circ} \mathrm{C}$, the collagen 577 layer was washed gently in PBS and $1 \mathrm{ml}$ of cell suspension $\left(10^{5} \mathrm{cells} / \mathrm{ml}\right)$ was 578 added. Cells were incubated for $90 \mathrm{~min}$ at $37^{\circ} \mathrm{C}$ in $1 \% \mathrm{CO}_{2}$ incubator, then pre579 extracted with $0.5 \%$ Triton X-100 in 4\% paraformaldehyde in PBS during 90 s and 580 fixed in $4 \%$ paraformaldehyde in PBS for $20 \mathrm{~min}$. Cells were analyzed by 581 immunofluorescence staining with TKS5 and CTTN antibodies (Table S2), and z582 stacks of images were acquired as described above. For quantification of TKS5 583 associated with curvilinear invadopodia, 5 consecutive planes corresponding to the 584 plasma membrane in contact with collagen fibers were projected and surface 585 covered by TKS5 signal was determined using the thresholding command of Image 586 excluding regions $<8$ pixels (i.e. $<1 \mu \mathrm{m}$ ) to avoid non-invadopodial structures. Surface 587 covered by TKS5 was normalized to the total cell surface and values normalized to 588 control cells.

Quantification of pericellular collagenolysis. Cells were trypsinized and

590 resuspended in $0.2 \mathrm{ml}$ of $2.2 \mathrm{mg} / \mathrm{ml}$ ice-cold collagen I solution in $1 \mathrm{x} \mathrm{MEM}, \mathrm{pH} 7.5$ 591 buffer $\left(2.5 \times 10^{5}\right.$ cells $\left./ \mathrm{ml}\right)$. A $40 \mu$-drop of the cell suspension in collagen was added 
592 on a 18-mm diameter glass coverslip and collagen polymerization was induced by

593 incubation at $37^{\circ} \mathrm{C}$ for $30 \mathrm{~min}$. Complete medium was added, and the collagen-

594 embedded cells were incubated for $12 \mathrm{hrs}$ at $37^{\circ} \mathrm{C}$. Samples were fixed in $4 \%$

595 paraformaldehyde in PBS at $37^{\circ} \mathrm{C}$ for $30 \mathrm{~min}$, and incubated with Col $1-{ }^{3 / 4} \mathrm{C}$ antibody

$596(2.5 \mu \mathrm{g} / \mathrm{ml})$ for $2 \mathrm{hrs}$ at $4^{\circ} \mathrm{C}$, washed extensively with PBS and counterstained with

597 Cy3-conjugated anti-rabbit IgG antibodies and with DAPI. Image acquisition was

598 performed with an A1R Nikon confocal microscope with a 40× NA 1.3 oil objective

599 using high-sensitivity GaASP PMT detector and a $595 \pm 50 \mathrm{~nm}$ band-pass filter for

600 red fluorescence detection. Quantification of the degradation spots was performed as

601 previously described $^{8}$.

602 Electron microscopy. Cells were fixed with $2,5 \%$ glutaraldehyde in $0,1 \mathrm{M}$ cacodylate 603 buffer and processed for EPON embedding and ultrathin sections as described ${ }^{62}$. 604 For cryoimmunolabeling, cells were fixed in a mixture of $2 \%$ paraformaldehyde and $6050.125 \%$ glutaraldehyde in a $0.1 \mathrm{M}$ phosphate buffer $\mathrm{pH} 7.4$ during $48 \mathrm{hrs}{ }^{62}$. Ultrathin 606 sections were prepared with an ultracryomicrotome Leica EM UC7 and underwent 607 single immunogold labeling with protein A conjugated to 10-nm diameter gold 608 particles (Cell Microscopy Center, Department of Cell Biology, Utrecht University). 609 Samples were analyzed with a Tecnai Spirit electron microscope (FEl Company, 610 Eindhoven Netherlands) and digital acquisitions were made with a QUEMESA CCD 611 camera (EMSIS GmbH, Münster, Germany).

612 Statistics and reproducibility. GraphPad Prism (GraphPad Software) was used for 613 statistical analysis. Sample size was chosen based on estimates from pilot 614 experiments and our published results such that appropriate statistical tests could 615 yield significant results. Data were tested for normal distribution using the 
616 D'Agostino-Pearson normality test and nonparametric tests were applied otherwise 617 as indicated in the figure legends. Statistical significance was defined as *, P<0.05;

$618{ }^{* *}, \mathrm{P}<0.01 ;{ }^{* *}, \mathrm{P}<0.001 ;{ }^{* * * *}, \mathrm{P}<0.0001$; ns, not significant. Relationships between 619 protein expression and distribution in tumors vs. normal adjacent tissues were 620 estimated using Two tailed Student's t-test and Kruskal-Wallis test (for links between 621 qualitative and quantitative parameters). Metastasis-free survival was determined as 622 the interval between initial diagnosis and detection of the first metastasis. Survival 623 distributions were estimated by the Kaplan-Meier method, and the significance of 624 differences between survival rates were ascertained with the log-rank test. The Cox 625 proportional hazards regression model was used to assess prognostic significance, 626 and the results are presented as hazard ratio (HR) and 95\% confidence interval $(\mathrm{Cl})$. 627 The following variables were included in the analysis: SBR grade, lymph node status, 628 macroscopic tumor size, PR status (all the classical variables with a $P$ value under 6290.10 in univariate analysis) and CORO1C or combined CORO1C and MT1-MMP 630 mRNA expression. For animal studies, no animals were excluded from analyses and 631 no blinding procedure was used.

633 See Supplementary Materials and Methods for additional cell lines, western blot 634 analysis, patient cohort for mRNA analysis, RT-qPCR analysis, patient biopsies for 635 western blot analysis, Immunohistochemistry analysis of human breast biopsies, 636 Mammary fat pad and intraductal injections, Mammary imaging window surgical 637 implantation and near-infrared multiphoton microscopy of mammary tumor 638 xenografts. 
641

6421 Rowe RG, Weiss SJ. Breaching the basement membrane: who, when and 643 how? Trends Cell Biol 2008; 18: 560-574.

644

6452 Lodillinsky C, Infante E, Guichard A, Chaligne R, Fuhrmann L, Cyrta J et al. 646 p63/MT1-MMP axis is required for in situ to invasive transition in basal-like breast 647 cancer. Oncogene 2016; 35: 344-357.

648

6493 Hotary K, Li XY, Allen E, Stevens SL, Weiss SJ. A cancer cell metalloprotease 650 triad regulates the basement membrane transmigration program. Genes Dev 2006; 651 20: 2673-2686.

652

6534 Wolf K, Wu YI, Liu Y, Geiger J, Tam E, Overall C et al. Multi-step pericellular 654 proteolysis controls the transition from individual to collective cancer cell invasion. 655 Nat Cell Biol 2007; 9: 893-904.

656

6575 Linder S, Wiesner C, Himmel M. Degrading devices: invadosomes in 658 proteolytic cell invasion. Annu Rev Cell Dev Biol 2011; 27: 185-211.

659 
6606 Artym VV, Zhang Y, Seillier-Moiseiwitsch F, Yamada KM, Mueller SC.

661 Dynamic interactions of cortactin and membrane type 1 matrix metalloproteinase at 662 invadopodia: defining the stages of invadopodia formation and function. Cancer Res 663 2006; 66: 3034-3043.

664

6657 Steffen A, Le Dez G, Poincloux R, Recchi C, Nassoy P, Rottner K et al. MT1666 MMP-dependent invasion is regulated by TI-VAMP/VAMP7. Curr Biol 2008; 18: 926667931.

668

6698 Monteiro P, Rosse C, Castro-Castro A, Irondelle M, Lagoutte E, Paul670 Gilloteaux $\mathrm{P}$ et al. Endosomal WASH and exocyst complexes control exocytosis of 671 MT1-MMP at invadopodia. J Cell Biol 2013; 203: 1063-1079.

672

6739 Gligorijevic B, Bergman A, Condeelis J. Multiparametric classification links 674 tumor microenvironments with tumor cell phenotype. PLoS Biol 2014; 12: e1001995.

675

67610 Leong HS, Robertson AE, Stoletov K, Leith SJ, Chin CA, Chien AE et al. 677 Invadopodia are required for cancer cell extravasation and are a therapeutic target 678 for metastasis. Cell Rep 2014; 8: 1558-1570.

679 
68011 Yamaguchi H, Lorenz M, Kempiak S, Sarmiento C, Coniglio S, Symons M et 681 al. Molecular mechanisms of invadopodium formation: the role of the N-WASP682 Arp2/3 complex pathway and cofilin. J Cell Biol 2005; 168: 441-452.

683

68412 Ayala I, Baldassarre M, Giacchetti G, Caldieri G, Tete S, Luini A et al. Multiple 685 regulatory inputs converge on cortactin to control invadopodia biogenesis and 686 extracellular matrix degradation. J Cell Sci 2008; 121: 369-378.

687

68813 Weaver AM, Karginov AV, Kinley AW, Weed SA, Li Y, Parsons JT et al. 689 Cortactin promotes and stabilizes Arp2/3-induced actin filament network formation. 690 Curr Biol 2001; 11: 370-374.

691

69214 Siton O, Ideses Y, Albeck S, Unger T, Bershadsky AD, Gov NS et al. Cortactin 693 releases the brakes in actin- based motility by enhancing WASP-VCA detachment 694 from Arp2/3 branches. Curr Biol 2011; 21: 2092-2097.

695

69615 Cai L, Makhov AM, Schafer DA, Bear JE. Coronin 1B antagonizes cortactin 697 and remodels Arp2/3-containing actin branches in lamellipodia. Cell 2008; 134: 828698842.

699 
70016 Chan KT, Creed SJ, Bear JE. Unraveling the enigma: progress towards

701 understanding the coronin family of actin regulators. Trends Cell Biol 2011; 21: 481702488.

703

70417 Abella JV, Galloni C, Pernier J, Barry DJ, Kjaer S, Carlier MF et al. Isoform 705 diversity in the Arp2/3 complex determines actin filament dynamics. Nat Cell Biol 706 2016; 18: 76-86.

707

70818 Humphries CL, Balcer HI, D'Agostino JL, Winsor B, Drubin DG, Barnes G et 709 al. Direct regulation of Arp2/3 complex activity and function by the actin binding 710 protein coronin. J Cell Biol 2002; 159: 993-1004.

711

71219 Foger N, Rangell L, Danilenko DM, Chan AC. Requirement for coronin 1 in T 713 lymphocyte trafficking and cellular homeostasis. Science 2006; 313: 839-842.

714

71520 Clark ES, Weaver AM. A new role for cortactin in invadopodia: regulation of 716 protease secretion. Eur J Cell Biol 2008; 87: 581-590.

71821 Clark ES, Brown B, Whigham AS, Kochaishvili A, Yarbrough WG, Weaver AM. 719 Aggressiveness of HNSCC tumors depends on expression levels of cortactin, a gene 720 in the 11q13 amplicon. Oncogene 2009; 28: 431-444. 
72222 Mader CC, Oser M, Magalhaes MA, Bravo-Cordero JJ, Condeelis J, Koleske

723 AJ et al. An EGFR-Src-Arg-cortactin pathway mediates functional maturation of 724 invadopodia and breast cancer cell invasion. Cancer Res 2011; 71: 1730-1741.

725

72623 Rosse C, Lodillinsky C, Fuhrmann L, Nourieh M, Monteiro P, Irondelle M et al.

727 Control of MT1-MMP transport by atypical PKC during breast-cancer progression.

728 Proc Natl Acad Sci U S A 2014; 111: E1872-1879.

729

73024 Molinie N, Gautreau A. The Arp2/3 Regulatory System and Its Deregulation in 731 Cancer. Physiol Rev 2018; 98: 215-238.

732

73325 Wu L, Peng CW, Hou JX, Zhang YH, Chen C, Chen LD et al. Coronin-1C is a 734 novel biomarker for hepatocellular carcinoma invasive progression identified by 735 proteomics analysis and clinical validation. J Exp Clin Cancer Res 2010; 29: 17.

736

73726 Ren G, Tian Q, An Y, Feng B, Lu Y, Liang J et al. Coronin 3 promotes gastric 738 cancer metastasis via the up-regulation of MMP-9 and cathepsin K. Molecular cancer 739 [electronic resource] 2012; 11: 67. 
74127 Liu C, Zhang S, Wang Q, Zhang X. Tumor suppressor miR-1 inhibits tumor 742 growth and metastasis by simultaneously targeting multiple genes. Oncotarget 2017;

743 8: 42043-42060.

744

74528 Thal D, Xavier CP, Rosentreter A, Linder S, Friedrichs B, Waha A et al.

746 Expression of coronin-3 (coronin-1C) in diffuse gliomas is related to malignancy. $\mathrm{J}$ 747 Pathol 2008; 214: 415-424.

748

74929 Ziemann A, Hess S, Bhuwania R, Linder S, Kloppenburg P, Noegel AA et al.

750 CRN2 enhances the invasiveness of glioblastoma cells. Neuro Oncol 2013; 15: 548751561.

752

75330 Hoshino D, Kirkbride KC, Costello K, Clark ES, Sinha S, Grega-Larson N et al. 754 Exosome secretion is enhanced by invadopodia and drives invasive behavior. Cell 755 Rep 2013; 5: 1159-1168.

756

75731 Macpherson IR, Rainero E, Mitchell LE, van den Berghe PV, Speirs C, 758 Dozynkiewicz MA et al. CLIC3 controls recycling of late endosomal MT1-MMP and 759 dictates invasion and metastasis in breast cancer. J Cell Sci 2014; 127: 3893-3901. 
76132 Marchesin V, Castro-Castro A, Lodillinsky C, Castagnino A, Cyrta J, Bonsang-

762 Kitzis $\mathrm{H}$ et al. ARF6-JIP3/4 regulate endosomal tubules for MT1-MMP exocytosis in 763 cancer invasion. J Cell Biol 2015; 211: 339-358.

764

76533 Castro-Castro A, Marchesin V, Monteiro P, Lodillinsky C, Rosse C, Chavrier P.

766 Cellular and Molecular Mechanisms of MT1-MMP-Dependent Cancer Cell Invasion.

767 Annu Rev Cell Dev Biol 2016; 32: 555-576.

768

76934 Gomez TS, Billadeau DD. A FAM21-containing WASH complex regulates 770 retromer-dependent sorting. Dev Cell 2009; 17: 699-711.

771

77235 Derivery E, Sousa C, Gautier JJ, Lombard B, Loew D, Gautreau A. The Arp2/3 773 activator WASH controls the fission of endosomes through a large multiprotein 774 complex. Dev Cell 2009; 17: 712-723.

775

77636 Holtta-Vuori M, Vainio S, Kauppi M, Van Eck M, Jokitalo E, Ikonen E. 777 Endosomal actin remodeling by coronin-1A controls lipoprotein uptake and 778 degradation in macrophages. Circ Res 2012; 110: 450-455.

78037 Kirkbride KC, Hong NH, French CL, Clark ES, Jerome WG, Weaver AM. 781 Regulation of late endosomal/lysosomal maturation and trafficking by cortactin 782 affects Golgi morphology. Cytoskeleton (Hoboken) 2012; 69: 625-643. 
78438 Sinha S, Hoshino D, Hong NH, Kirkbride KC, Grega-Larson NE, Seiki M et al. 785 Cortactin promotes exosome secretion by controlling branched actin dynamics. J Cell 786 Biol 2016; 214: 197-213.

787

78839 Smith SE, Mellor P, Ward AK, Kendall S, McDonald M, Vizeacoumar FS et al. 789 Molecular characterization of breast cancer cell lines through multiple omic 790 approaches. Breast Cancer Res 2017; 19: 65.

791

79240 Juin A, Billottet C, Moreau V, Destaing O, Albiges-Rizo C, Rosenbaum J et al. 793 Physiological type I collagen organization induces the formation of a novel class of 794 linear invadosomes. Molecular biology of the cell 2012; 23: 297-309.

795

79641 Chan KT, Roadcap DW, Holoweckyj N, Bear JE. Coronin 1C harbours a 797 second actin-binding site that confers co-operative binding to F-actin. Biochem $\mathrm{J}$ $798 \quad 2012 ; 444: 89-96$.

799

80042 Cai L, Makhov AM, Bear JE. F-actin binding is essential for coronin 1B 801 function in vivo. J Cell Sci 2007; 120: 1779-1790.

802 
80343 Behbod F, Kittrell FS, LaMarca H, Edwards D, Kerbawy S, Heestand JC et al. 804 An intraductal human-in-mouse transplantation model mimics the subtypes of ductal 805 carcinoma in situ. Breast Cancer Res 2009; 11: R66.

806

80744 Mukherjee S, Maxfield FR. Lipid and cholesterol trafficking in NPC. Biochim 808 Biophys Acta 2004; 1685: 28-37.

809

81045 Liscum L, Faust JR. The intracellular transport of low density lipoprotein-

811 derived cholesterol is inhibited in Chinese hamster ovary cells cultured with 3-beta812 [2-(diethylamino)ethoxy]androst-5-en-17-one. J Biol Chem 1989; 264: 11796-11806.

813

81446 Wang J, Tsouko E, Jonsson P, Bergh J, Hartman J, Aydogdu E et al. miR-206 815 inhibits cell migration through direct targeting of the actin-binding protein coronin $1 \mathrm{C}$ 816 in triple-negative breast cancer. Mol Oncol 2014; 8: 1690-1702.

817

81847 McGowan PM, Duffy MJ. Matrix metalloproteinase expression and outcome in 819 patients with breast cancer: analysis of a published database. Ann Oncol 2008; 19: $820 \quad 1566-1572$.

821

82248 Perentes JY, Kirkpatrick ND, Nagano S, Smith EY, Shaver CM, Sgroi D et al. 823 Cancer cell-associated MT1-MMP promotes blood vessel invasion and distant 824 metastasis in triple-negative mammary tumors. Cancer Res 2011; 71: 4527-4538. 
82649 Rosentreter A, Hofmann A, Xavier CP, Stumpf M, Noegel AA, Clemen CS.

827 Coronin 3 involvement in F-actin-dependent processes at the cell cortex. Exp Cell 828 Res 2007; 313: 878-895.

829

83050 Yu X, Zech T, McDonald L, Gonzalez EG, Li A, Macpherson I et al. N-WASP 831 coordinates the delivery and F-actin-mediated capture of MT1-MMP at invasive 832 pseudopods. J Cell Biol 2012; 199: 527-544.

833

83451 Cai L, Marshall TW, Uetrecht AC, Schafer DA, Bear JE. Coronin 1B 835 coordinates Arp2/3 complex and cofilin activities at the leading edge. Cell 2007; 128: $836 \quad 915-929$.

837

83852 Eckert MA, Lwin TM, Chang AT, Kim J, Danis E, Ohno-Machado L et al. 839 Twist1-induced invadopodia formation promotes tumor metastasis. Cancer Cell 2011; 840 19: 372-386.

841

84253 Puthenveedu MA, Lauffer B, Temkin P, Vistein R, Carlton P, Thorn K et al. 843 Sequence-dependent sorting of recycling proteins by actin-stabilized endosomal 844 microdomains. Cell 2010; 143: 761-773. 
84654 Gomez TS, Gorman JA, de Narvajas AA, Koenig AO, Billadeau DD. 847 Trafficking defects in WASH-knockout fibroblasts originate from collapsed endosomal 848 and lysosomal networks. Mol Biol Cell 2012; 23: 3215-3228.

849

85055 Hong NH, Qi A, Weaver AM. PI(3,5)P2 controls endosomal branched actin 851 dynamics by regulating cortactin-actin interactions. J Cell Biol 2015; 210: 753-769.

852

85356 Willett R, Martina JA, Zewe JP, Wills R, Hammond GRV, Puertollano R. TFEB 854 regulates lysosomal positioning by modulating TMEM55B expression and JIP4 855 recruitment to lysosomes. Nat Commun 2017; 8: 1580.

856

85757 Sakurai-Yageta M, Recchi C, Le Dez G, Sibarita JB, Daviet L, Camonis J et al. 858 The interaction of IQGAP1 with the exocyst complex is required for tumor cell 859 invasion downstream of Cdc42 and RhoA. J Cell Biol 2008; 181: 985-998.

860

86158 Marchesin V, Montagnac G, Chavrier P. ARF6 promotes the formation of Rac1 862 and WAVE-dependent ventral F-actin rosettes in breast cancer cells in response to 863 epidermal growth factor. PLoS One 2015; 10: e0121747.

864

86559 Sibarita JB. Deconvolution microscopy. Advances in biochemical 866 engineering/biotechnology 2005; 95: 201-243. 
86860 Lamprecht MR, Sabatini DM, Carpenter AE. CellProfiler: free, versatile 869 software for automated biological image analysis. BioTechniques 2007; 42: 71-75.

870

87161 Castro-Castro A, Janke C, Montagnac G, Paul-Gilloteaux P, Chavrier P. 872 ATAT1/MEC-17 acetyltransferase and HDAC6 deacetylase control a balance of 873 acetylation of alpha-tubulin and cortactin and regulate MT1-MMP trafficking and 874 breast tumor cell invasion. Eur J Cell Biol 2012; 91: 950-960.

875

87662 Hurbain I, Romao M, Bergam P, Heiligenstein X, Raposo G. Analyzing 877 Lysosome-Related Organelles by Electron Microscopy. Methods Mol Biol 2017; 878 1594: 43-71.

879

880

881

882 
883 Acknowledgments. The authors greatly acknowledge the Breast Cancer Study 884 Group and patients of Institut Curie for breast tumor samples. They thank the Cell 885 and Tissue Imaging facility (PICT-IBiSA) and Nikon Imaging Centre, Institut Curie, 886 member of the French National Research Infrastructure France-Biolmaging (ANR10887 INBS-04) for help with image acquisition and Dr Jakko van Rheenen for help with the 888 mammary window and intravital imaging. A.C. was supported by a grant from 889 Worldwide Cancer Research (Grant $16-1235$ to P.C.), A.C.C. by an EMBO Long 890 Term postdoctoral fellowship and a grant from 'Institut National du Cancer' 891 (INCA_6521 to P.C.), A.G. by INVADE grant from ITMO Cancer (Call Systems 892 Biology 2012) to P.C., S.A.G. by a grant from INCa (INCa 2014-11/474/AB-SD to 893 P.C.). and A.A.N. and C.S.C by a grant from the German Research Foundation (DFG 894 NO 113/22-2). The intravital imaging was supported by the Fondation pour la 895 Recherche Médicale (FRM N DGE20111123020), the Cancerople-IdF (n²012-2896 EML-04-IC-1), InCA (Cancer National Institute, $n^{\circ}$ 2011-1-LABEL-IC-4) and SiRIC 897 (INCa-DGOS- 4654). This work was supported by grants from 'Institut National $d u$ 898 Cancer' (INCa 2014-11/474/AB-SD and INCA_6521), Worldwide Cancer Research 899 (Grant 16-1235) and 'Equipe labellisée 2015' from 'Ligue Nationale contre le Cancer' 900 to P.C. 


\section{Author contributions}

903 AC carried out invadopodia formation and collagen degradation assays with the help 904 of AZM and characterized the effects of CORO1C depletion on endolysosomal 905 morphology and position. ACC who initiated the study, generated the cell lines stably 906 expressing ${ }^{\mathrm{GFP}}$ CORO1C constructs and analyzed CORO1B and $-1 \mathrm{C}$ expression in 907 lysates of breast cancer cell lines provided by TD. LF and AVS generated the breast 908 cancer TMA, performed CORO1C IHC labeling and scored the breast tumor TMA for 909 CORO1C expression with the help of CEK. SV and IB generated the invasive breast 910 cancer cohort and performed RT-qPCR and multivariate analyses. MI performed 911 multicellular spheroid invasion assays, analyzed the phenotype of cells silenced for 912 cortactin and performed intravital imaging. AG, CL and SAG carried out mammary 913 gland injections and analyzed tumor xenografts. MR and GR performed electron 914 microscopy analyses. JEB, AAN and CSC provided essential reagents. PC 915 supervised the study and wrote the manuscript with contribution of all authors. 


\section{Figure Legends}

918 Figure 1. Patients displaying high CORO1C and MT1-MMP mRNA expression

919 have poor outcomes in breast cancer. (A) Whole-cell lysates from human breast

920 carcinoma cell lines and Jurkat T cells were normalized for protein concentration and

921 immunoblotted with indicated antibodies. Stratification of breast cancer cell lines in

922 Normal-like, TNBC, Luminal and HER2 subtypes was according to ${ }^{39}$. (B-E)

923 Expression of the indicated proteins in the different cell lines divided in four subtypes

924 was normalized to GAPDH level. Protein levels were analyzed in 2 to 3 experiments

925 for each protein in each cell line and plots correspond to average expression levels \pm

926 SEM in each subtype. (F) Expression of the indicated genes was analyzed by RT-

927 qPCR in 446 mRNA tumor samples. Patients exhibiting high CORO1C mRNA have

928 reduced MFS (log-rank test using optimal cutoff of $1.52 ; p=0.00082$ ). Number of

929 patients in each subgroup is indicated in parenthesis. (G) Correlation between

930 indicated gene transcripts was analyzed using the Spearman's rank correlation test

931 (in bold type). $P$ values are in italics. (H) Patients exhibiting both high CORO1C and

932 MT1-MMP mRNAs have reduced MFS (log-rank test using optimal cut-off of 1.52 for

933 CORO1C and 1.30 for MT1-MMP; $P=0.00048)$.

934 Figure 2. Increased CORO1C expression in invasive breast cancers. (A, B)

935 CORO1C immunohistochemistry staining on sections of human invasive breast

936 cancer (IBC) tissue microarray showing adjacent non-neoplastic tissue (Normal) and

937 representative TNBC and HER2+ breast cancers. (C, D) Semi-quantitative analysis

938 of CORO1C protein expression by the $\mathrm{H}$-score method comparing adjacent breast

939 epithelial tissues and carcinoma tissues (C) or in the different subtypes (D). Two

940 tailed t-test (C); Kruskal-Wallis test (D). 
941 Figure 3. CORO1C is required for 3D invasion and invadopodia function. (A)

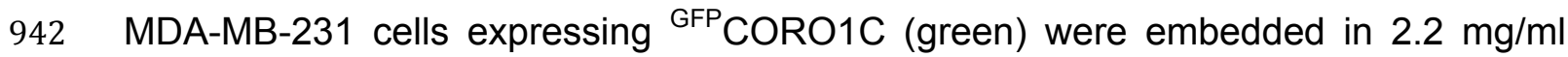

943 fluorescent type I collagen (magenta) and polymerization was induced at neutral $\mathrm{pH}$

944 at $37^{\circ} \mathrm{C}$. Cells were fixed after $12-16 \mathrm{~h}$ and stained for cortactin (red). The nucleus is

945 stained with DAPI (blue). The image is a single optical plane from a z-stack

946 (Supplementary Movie S1). The low magnification inset is a projection of the z-stack

947 of images (collagen is omitted). Inset 1 shows separated channels corresponding to

948 the boxed region. (B) Cells in 3D collagen (magenta) stained for cleaved collagen

949 neoepitope (Col $1-{ }^{3 / 4} \mathrm{C}$ antibody, yellow) and F-actin (blue). DAPI-stained nuclei are

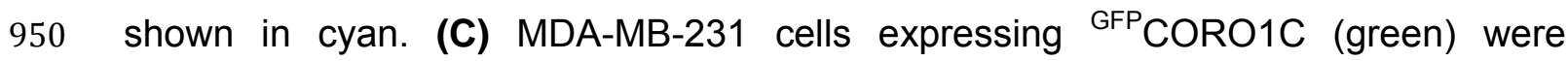

951 incubated on a thick layer of fibrous type I collagen (magenta). Curvilinear

952 invadopodia forming in association with collagen fibrils (cyan) were labeled for TKS5

953 (red). Insets show separated channels corresponding to the boxed region. (D)

954 Phalloidin-labeled multicellular spheroids of MDA-MB-231 cells expressing GFP or

$955{ }^{\text {GFP }}$ CORO1C after 2 days in 3D collagen I (T2). Insets show spheroids immediately

956 after embedding in collagen (TO). (E) Mean invasion area of multicellular spheroids at

957 T2 in the presence or absence of GM6001 normalized to mean spheroid area at T0 \pm

958 SEM. n, spheroid number. Two-way ANOVA. (F) Quantification of invadopodia-

959 associated TKS5 signal in MDA-MB-231 cells expressing GFP, ${ }^{\text {GFPCORO1C or }}$

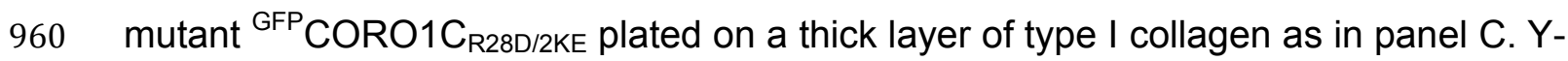

961 axis indicates TKS5 area normalized to total cell area and to mean value in MDA-

962 MB-231 cells (as percentage) \pm SEM. $n$, number of cells analyzed from three

963 independent experiments. Kruskal-Wallis test. (G) TKS5-positive invadopodia in

964 MDA-MB-231 cells knocked down for CORO1C, CORO1B or both as in panel F. $n$,

965 number of cells analyzed from five (siNT), three (siCORO1C ${ }^{\# 04}$ and ${ }^{\# 08}$ ) or two 
966 (siCORO1B and siCORO1B+C $\# 04$ ) independent experiments. (H) MDA-MB-231 cells 967 expressing CORO1B $^{\text {GFP }}$ (green) and MT1-MMP ${ }^{m C h}$ (red) plated on fibrous type I 968 collagen (magenta). CORO1B and MT1-MMP colocalize in invadopodia forming in 969 association with the collagen fibers (inset 1). CORO1B-positive puncta are also 970 visible on MT1-MMP endolysosomes (inset 2). (I) Representative images of 971 pericellular collagenolysis detected with Col1- ${ }^{3 / 4} \mathrm{C}$ antibody (black signal in the 972 inverted images). Nuclei were stained with DAPI (red). (J, K) Collagen degradation 973 measured by Col $1-{ }^{3 / 4} \mathrm{C}$ neoepitope staining by indicated cell populations normalized 974 to GFP-expressing (I) or siNT-treated (J) control cells \pm SEM; $n$, number of cells 975 analyzed from three independent experiments. Data were transformed using the log 976 transformation $y=\log (y)$ to make data conform to normality and analyzed using one977 way ANOVA.

Figure 4. In vivo association of CORO1C-positive invadopodia with ECM fibers. 979 (A-D) Near-infrared multiphoton microscopy images of tumor sections of 8-week-old 980 fat pad tumor xenografts from MDA-MB-231/ ${ }^{\text {GFP }}$ CORO1C (panels A to C) or MDA$981 \mathrm{MB}-231 /{ }^{\mathrm{GFP}} \mathrm{CORO} 1 \mathrm{C}_{\mathrm{R} 28 \mathrm{D} / 2 \mathrm{KE}}$ cells (panel D). Collagen fibers detected by second 982 harmonic generation (SHG, magenta). (G-I) Time-course of the growth and invasion 983 of MCF10DCIS.com/ ${ }^{\text {GFP }}$ CORO1C intraductal tumor xenograft monitored by near984 infrared multiphoton microscopy 5 to 7 -week post injection. All images are single 985 optical sections from z-stack except in panel $G$ and $H$, which show maximum 986 intensity projections of indicated optical planes. Insets are higher magnification of 987 boxed regions. White arrows point to accumulations of ${ }^{\mathrm{GFP}} \mathrm{CORO} 1 \mathrm{C}$ in association 988 with collagen fibers.

989 Figure 5. Altered MT1-MMP endosome positioning and morphology upon

990 CORO1C knockdown. (A) Still image from live cell confocal microscopy sequence 
991 of MDA-MB-231 cells expressing MT1-MMP ${ }^{m C h}$ and ${ }^{\text {GFPCORO1C (see }}$

992 Supplementary Movie S2). Inset is a high magnification of the boxed region. Bottom

993 row shows the fluorescence intensity profiles of MT1-MMP ${ }^{m C h}$ and ${ }^{\text {GFP }}$ CORO1C

994 along the dotted line. (B) High magnification of MDA-MB-231 cell expressing MT1-

$995 \mathrm{MMP}^{\mathrm{mCh}}$ and ${ }^{\mathrm{GFP}} \mathrm{CORO} 1 \mathrm{C}_{\mathrm{R} 28 \mathrm{D} / 2 \mathrm{KE}}$ as in panel $\mathrm{A}$. Fluorescence intensity profiles are

996 shown. (C) MDA-MB-231 cells expressing MT1-MMP ${ }^{m C h}$ (red) and ${ }^{\text {GFPCORO1C }}$

997 (green) stained for CTTN (blue). (D) Cryoimmunoelectron microscopy of MDA-MB-

998231 cells expressing GFPCORO1C. The micrograph shows the localization of

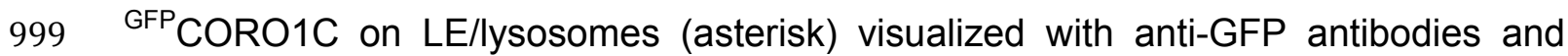
1000 secondary antibodies coupled to 10-nm diameter protein A-gold (arrows).

1001 Arrowheads point to ${ }^{\mathrm{GFP}}$ CORO1C associated with the cytosolic face of the plasma 1002 membrane. (E-H). Confocal fluorescence microscopy images of MDA-MB-231 cells 1003 expressing MT1-MMP ${ }^{\mathrm{mCh}}$ (red) superimposed with the phase-contrast image (grey). 1004 Cells are treated with indicated siRNAs. Cell contour is shown with dashed line. (I) 1005 Distribution of MT1-MMP ${ }^{m C h}$-positive endosomes in MDA-MB-231 cells treated with 1006 indicated siRNAs. Mean percentage of MT1-MMP-positive endosomes according to 1007 their cell center-to-cell periphery position \pm SEM from three independent 1008 experiments. (J) Proportion of MDA-MB-231 cells (\%) with juxtanuclear clustered or 1009 scattered MT1-MMPmCh endosome distribution from four to five independent 1010 experiments (Fig. S6C-E).

1011 Figure 6. Knockdown of CORO1C affects the distribution of MT1-MMP-positive 1012 endolysosomes and JIP4 and CTTN association. (A-C) Indirect 1013 immunofluorescence analysis of MDA-MB-231 cells expressing MT1-MMP ${ }^{\mathrm{mCh}}$ treated $^{\text {M }}$ 1014 with indicated siRNAs with CTTN (blue) and JIP4 (green) antibodies. DAPI-stained 1015 nuclei are shown in cyan. (D, E) CTTN (D) and JIP4 (E) association with the cytosolic 
1016 face of MT1-MMPmCh-positive endosomes analyzed based on images such as in 1017 panel A-C, compared with siNT-treated cells \pm SEM from three independent 1018 experiments. $\mathrm{n}$, number of cells analyzed for each population. (F-I) Electron 1019 microscopy of ultrathin sections of epon-embedded cells treated with indicated 1020 siRNAs. Notice the large aggregated endolysosomes in CORO1C-depleted cells 1021 (asterisks). N, nucleus; LE, endolysosome; m, mitochondria. 


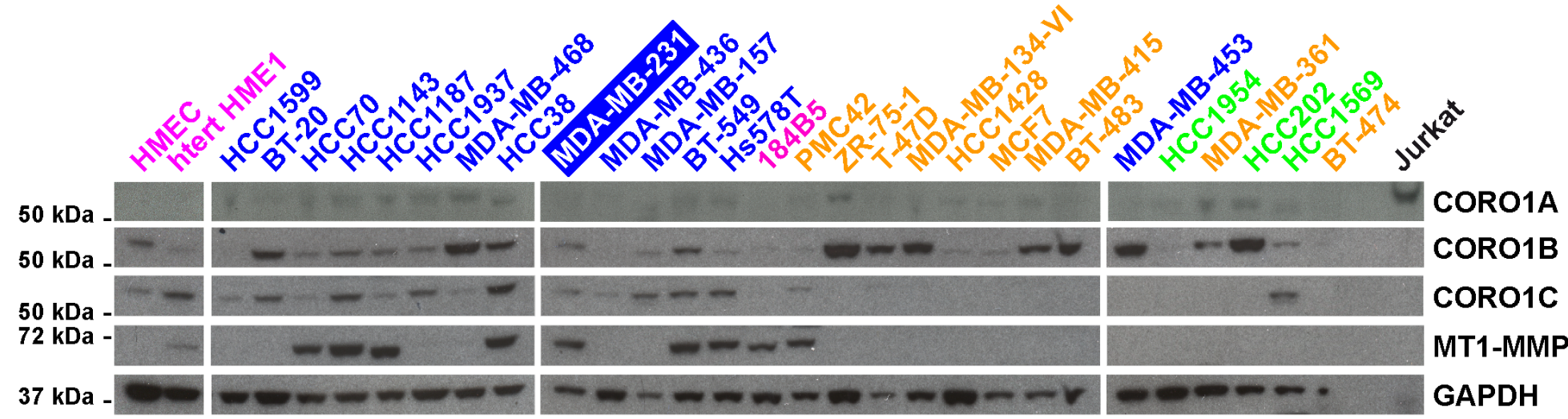

B

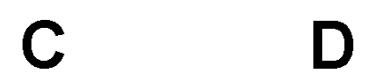

8

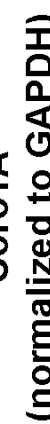

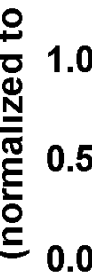

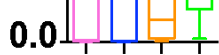

$+8020$

G

\begin{tabular}{lcccc}
\multicolumn{5}{c}{ CORO1B CORO1C MT1-MMP CTTN } \\
\hline CORO1B & 1 & & & \\
CORO1C & 0.312 & 1 & & \\
& $<1 \times 10^{-4}$ & & & \\
MT1-MMP & 0.162 & 0.448 & 1 & \\
& 0.00072 & $<1 \times 10^{-4}$ & & \\
CTTN & 0.555 & 0.249 & 0.133 & 1 \\
& $<1 \times 10^{-4}$ & $<1 \times 10^{-4}$ & 0.0051 & \\
\hline
\end{tabular}

Spearman correlation coefficients. $P$ values. $\mathrm{N}=446$.

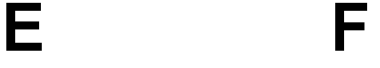

F
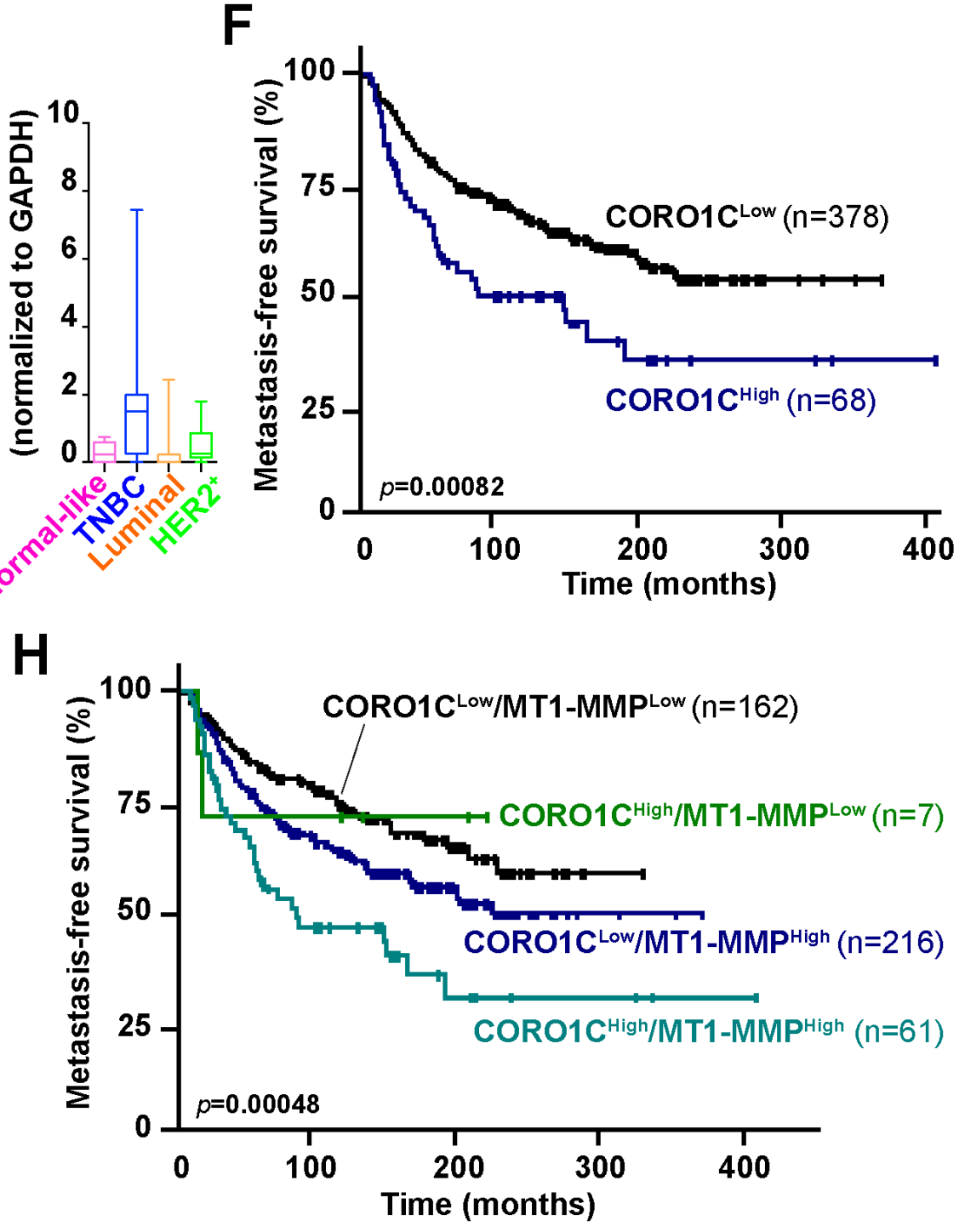

FIGURE 1 
A

Coro1C IHC
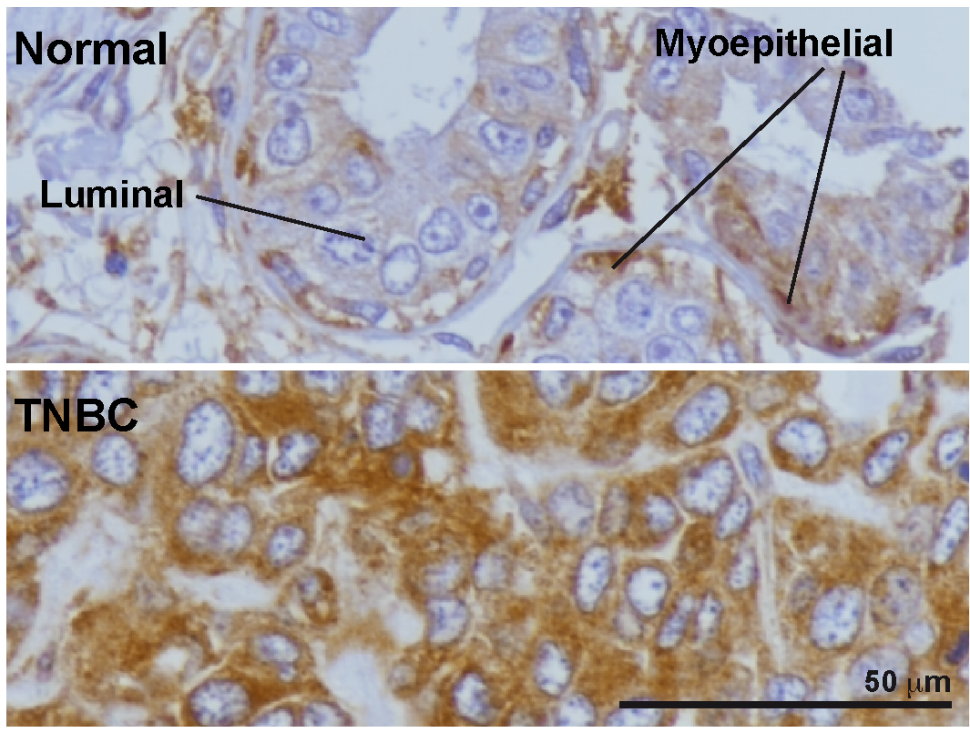

B
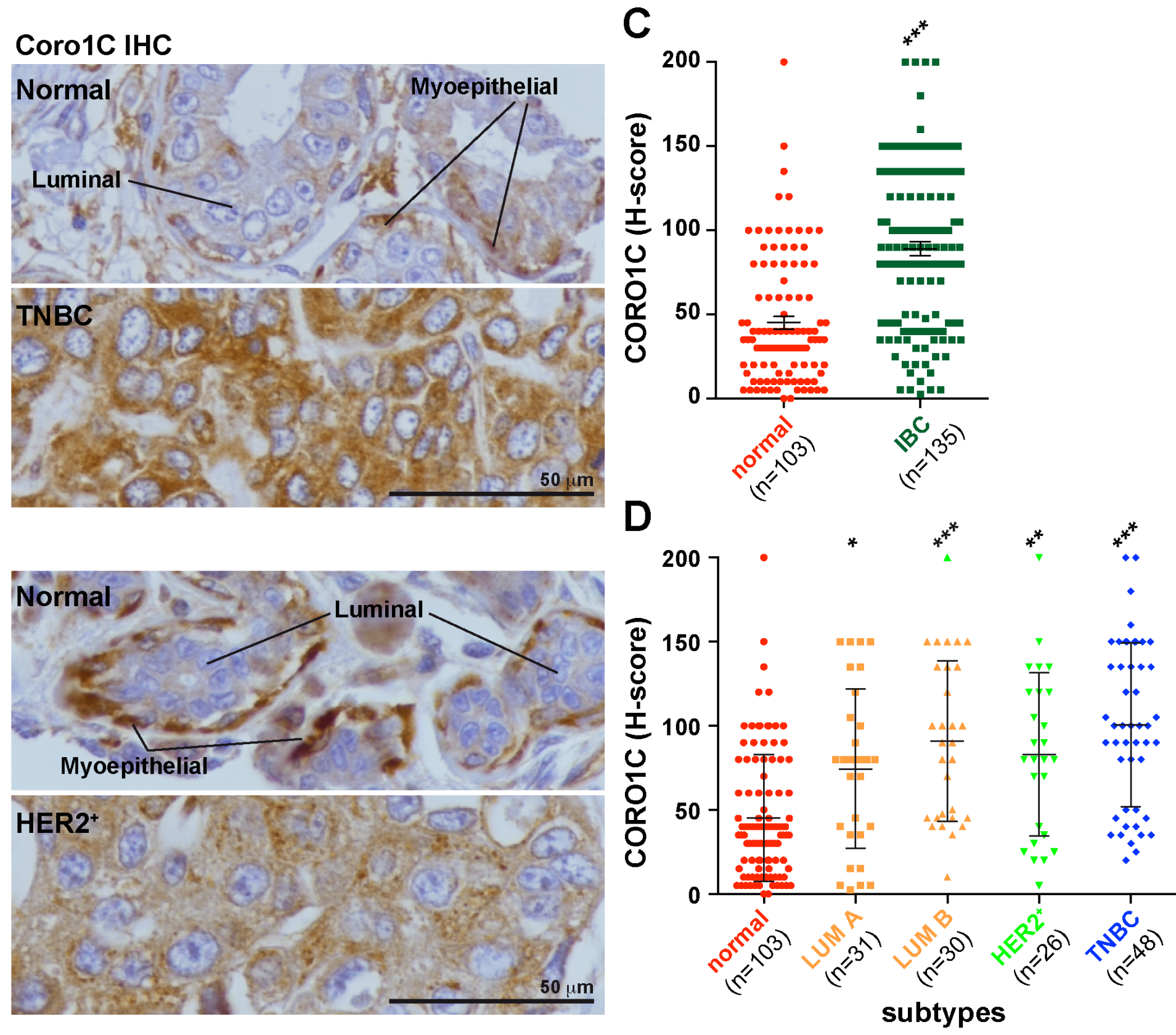

\section{FIGURE 2}


A

MDA-MB-231

GFPCORO1C/CTTN/3D Coll1

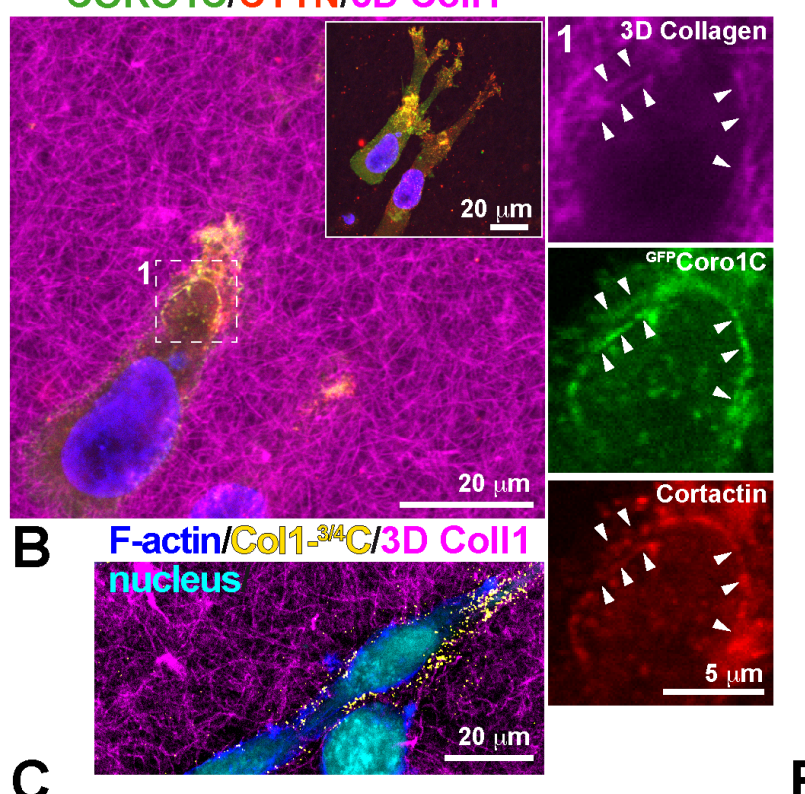

MDA-MB-231/GFPCORO1C
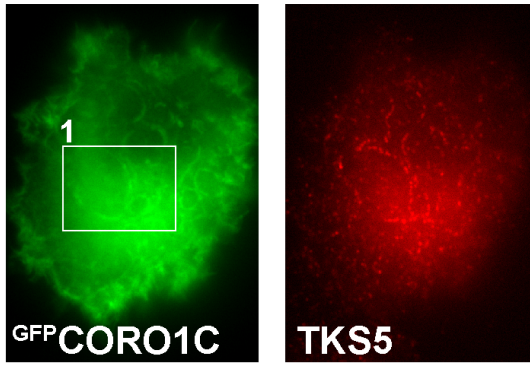

GFPCORO1C
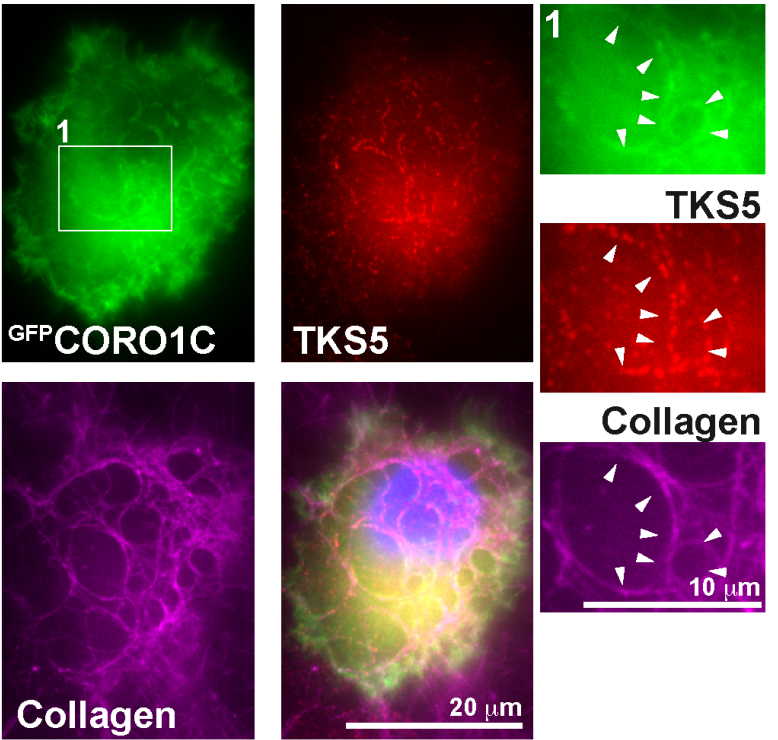

H CORO1B GFP/MT1-MIMPMCh/Coll1/nucleus
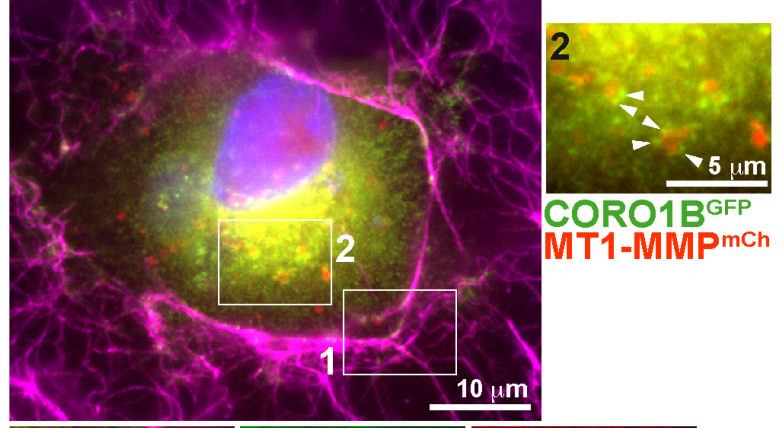

MT1-MMPmch

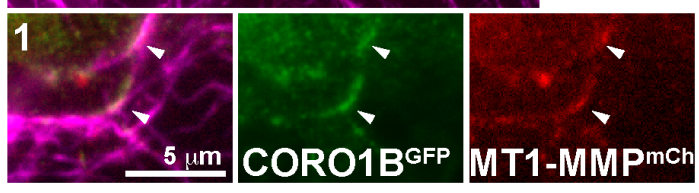

D
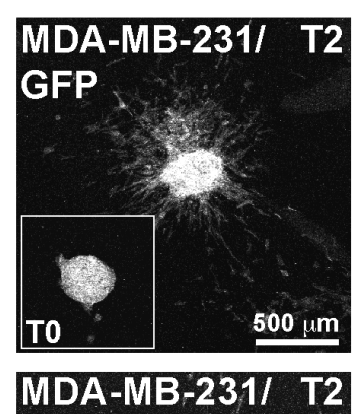

GFPCORO1C.

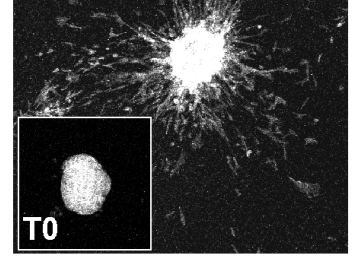

$\mathbf{F}$

G
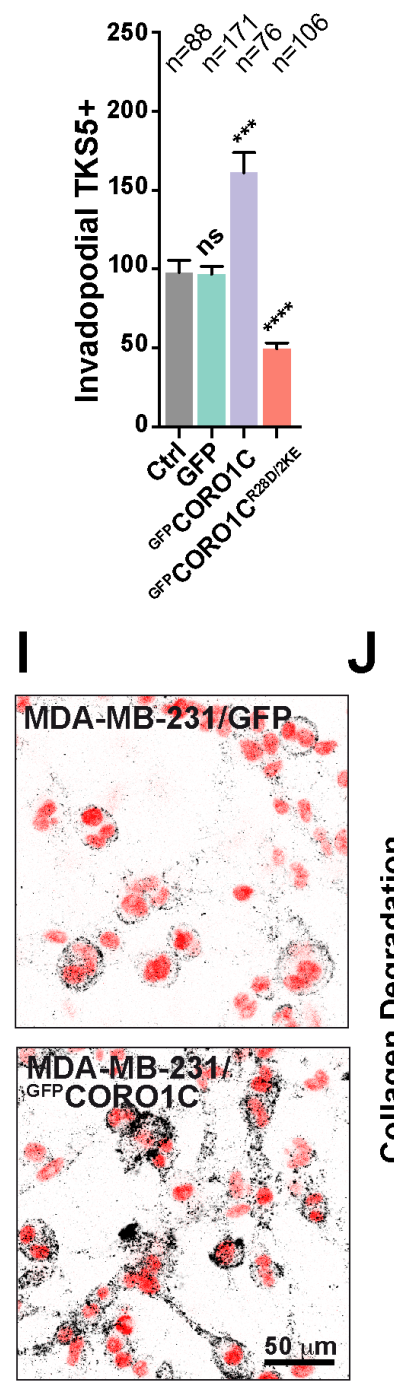

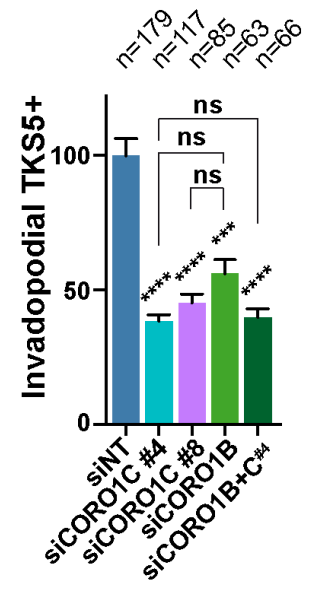

MDA-MB-231 GFP GFPCOR01C

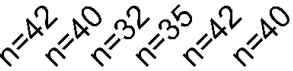
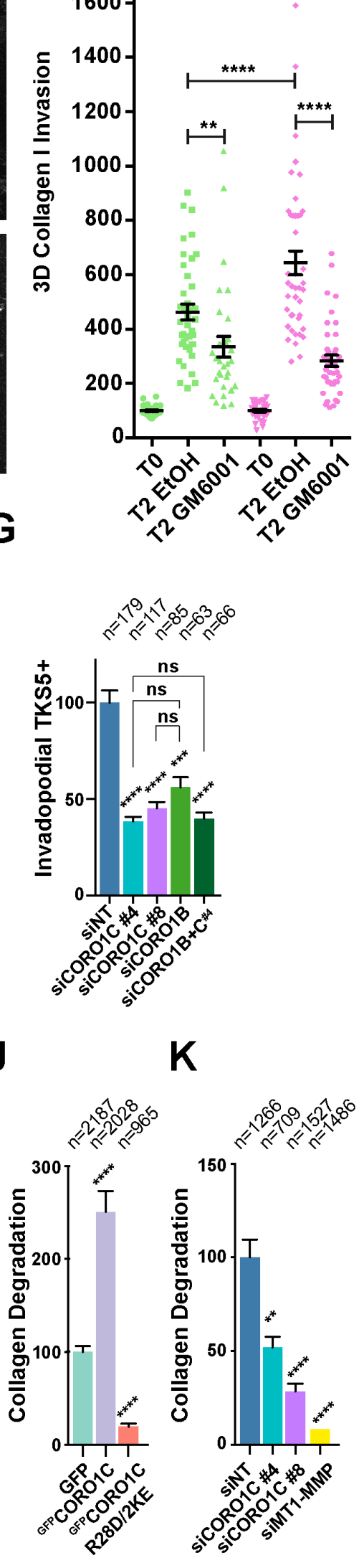

FIGURE 3 
Fat pad injection - MDA-MB-231//GFPCoro1C (8-week pi)
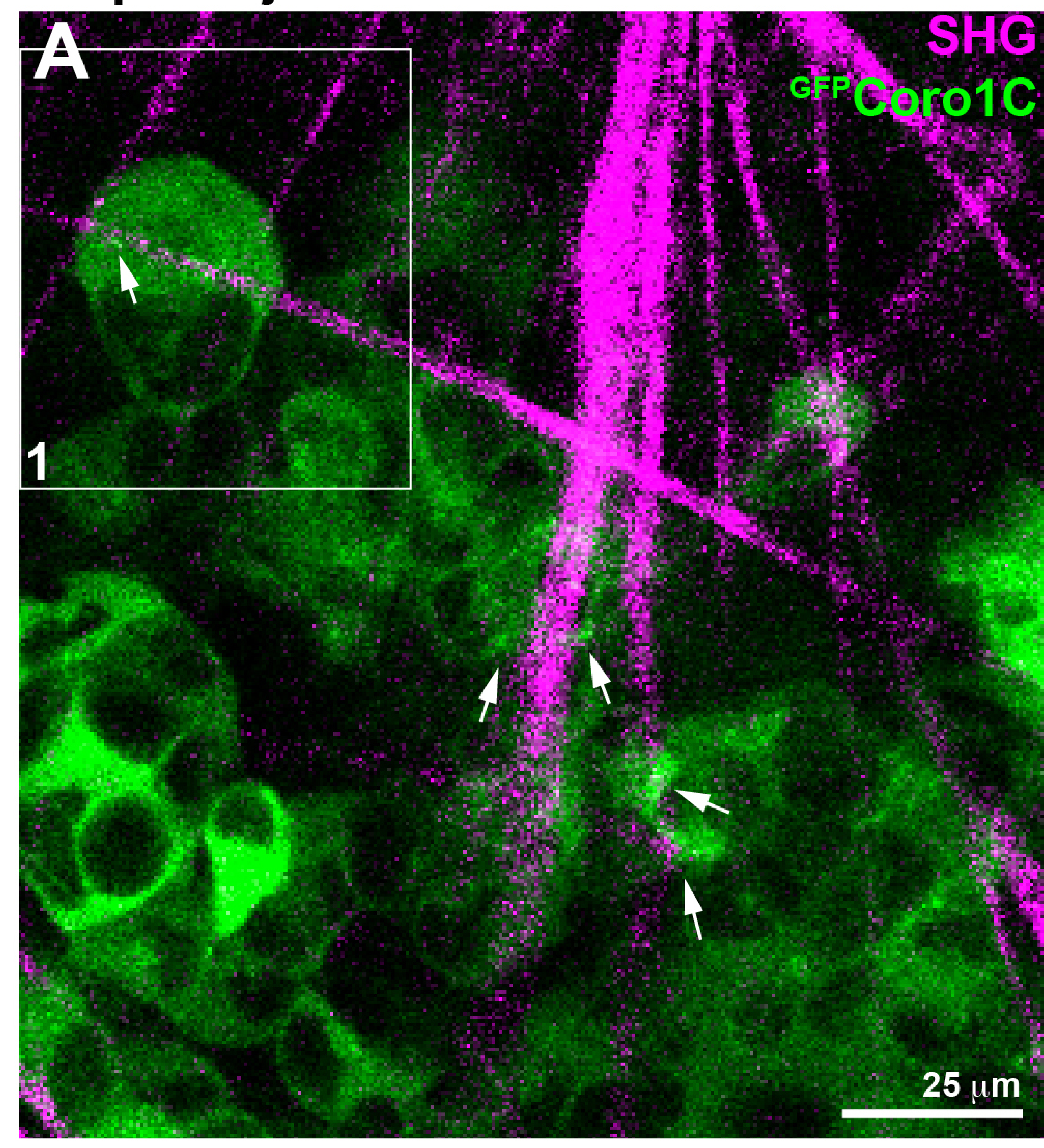
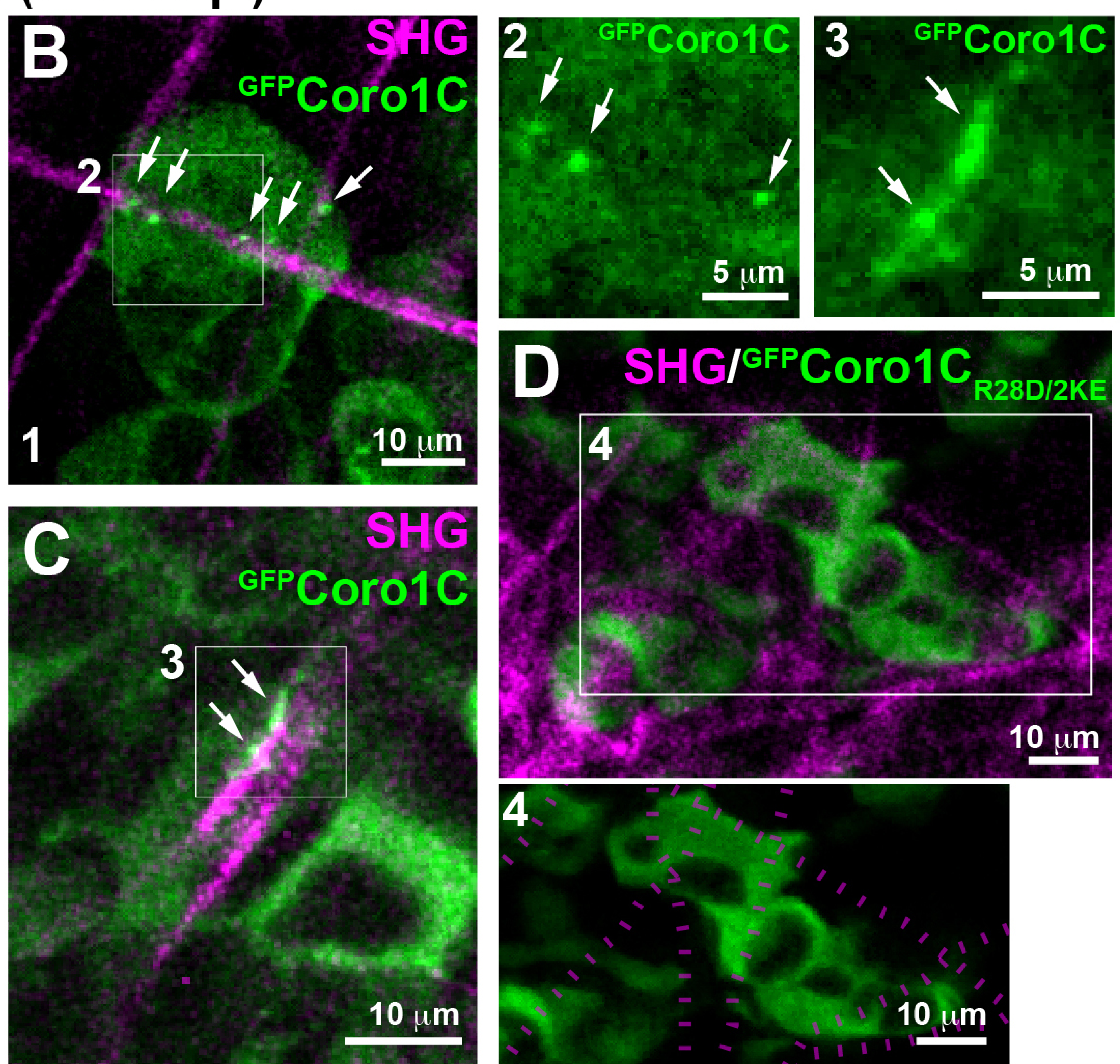

\section{Intraductal injection - MCF10DCIS.com/ ${ }^{\text {GFPC }}$ Coro1C}

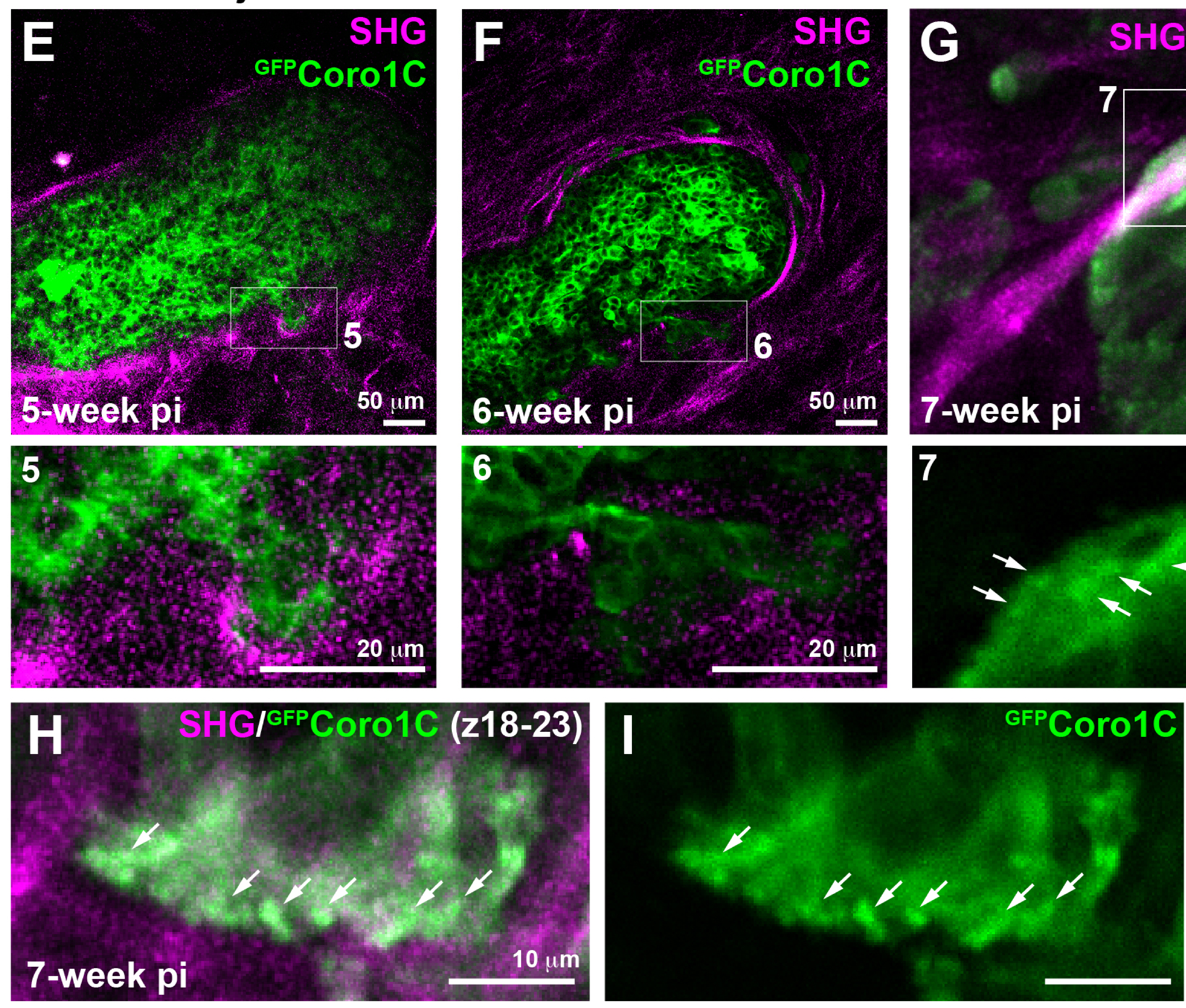

\section{FIGURE 4}


A

MT1-MMPmch/GFP CORO1C

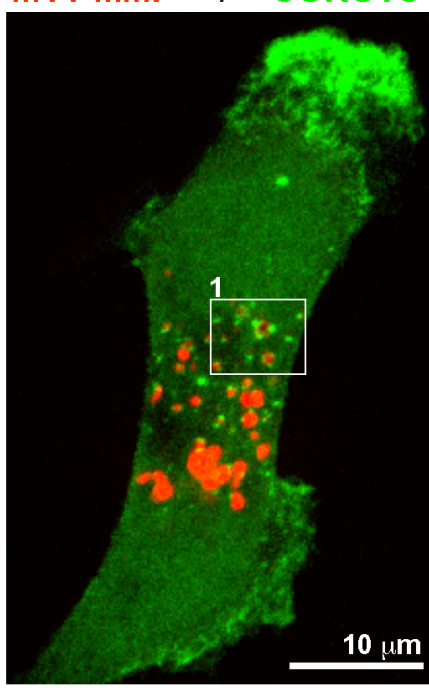

D
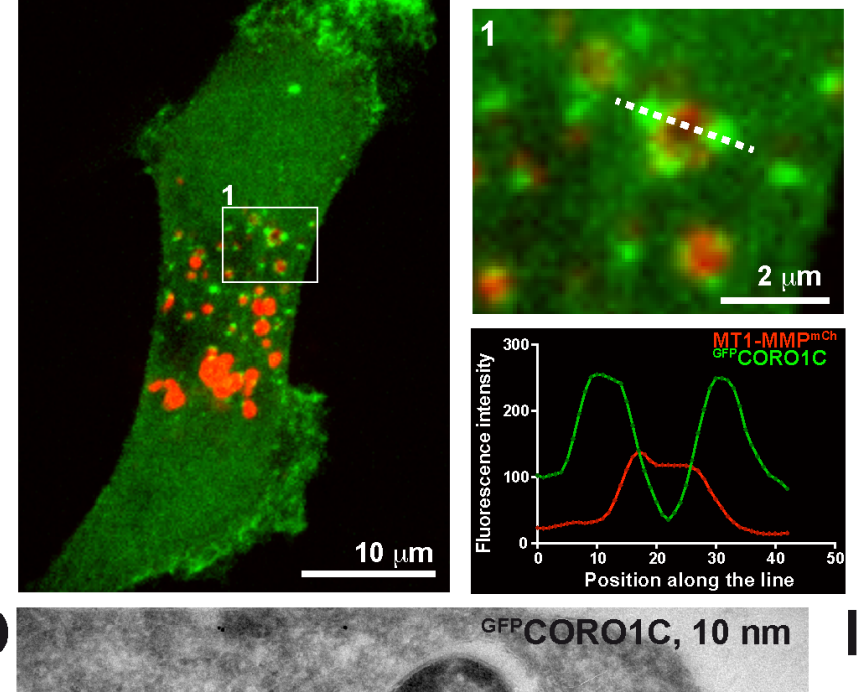

GFPCORO1C, $10 \mathrm{~nm}$

1

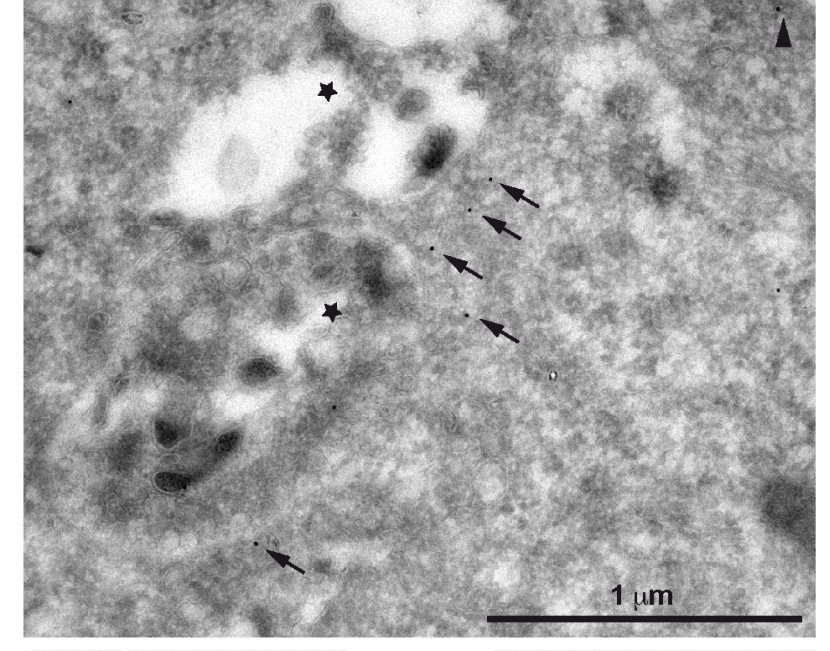

E

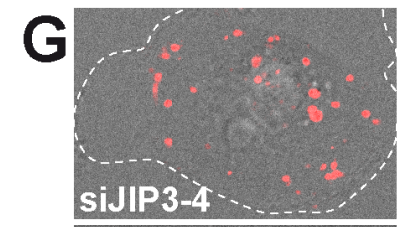

$\mathbf{F}$

$$
\text { SiNT }
$$

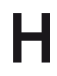

sijip3-4 +

sicóro1e \#4

FIGURE 5
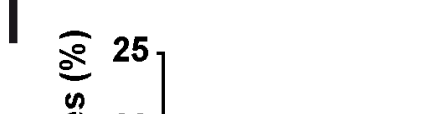

\& 20

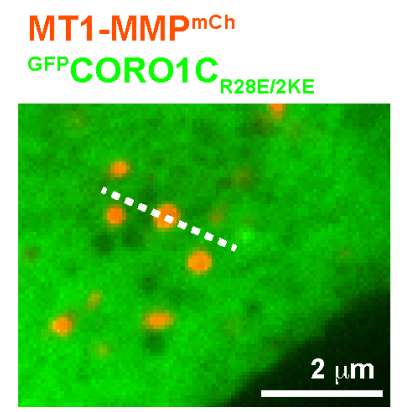

C MT1-MMPmCh/GFPCORO1Cl CTTN
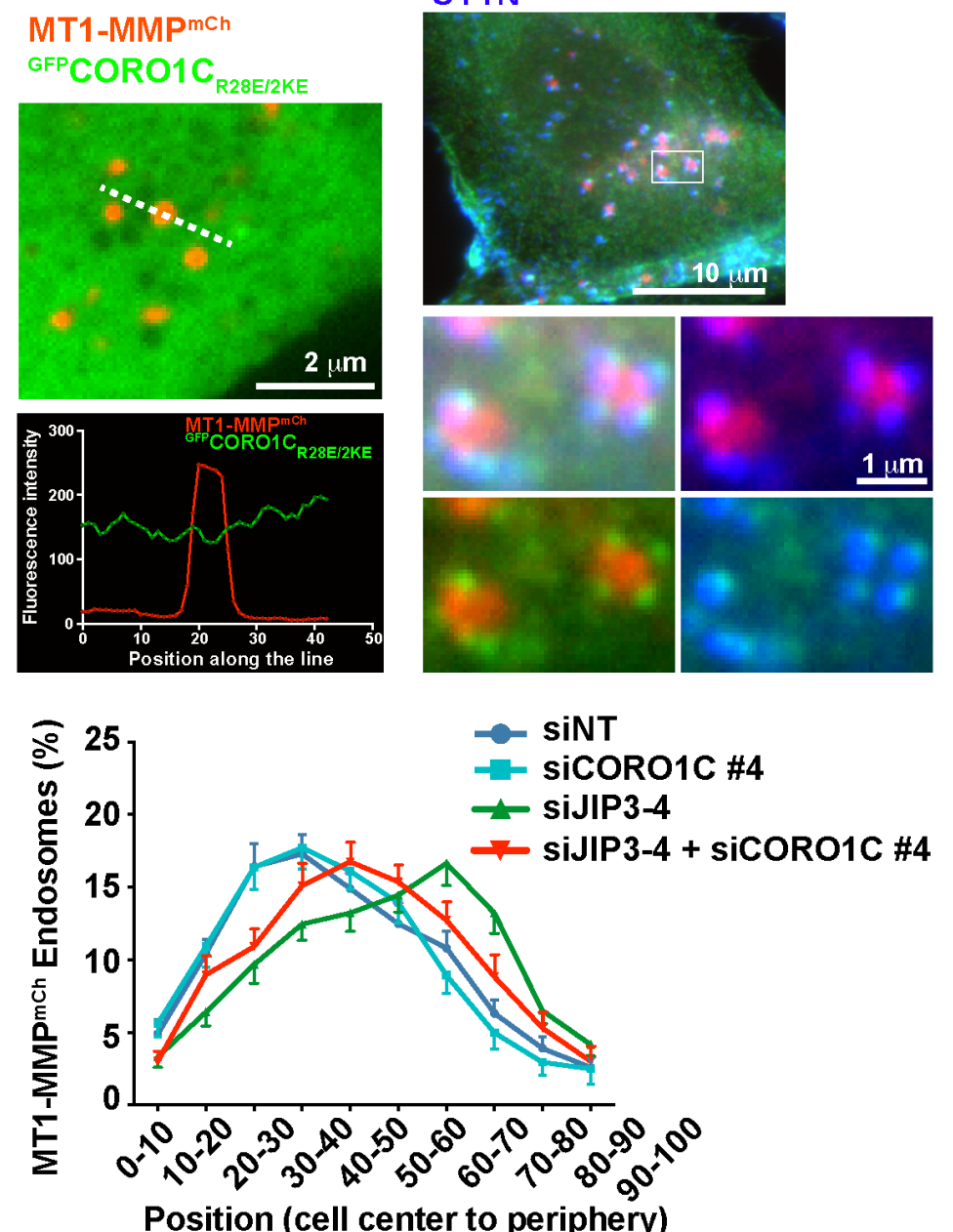

$\sqrt{ }$

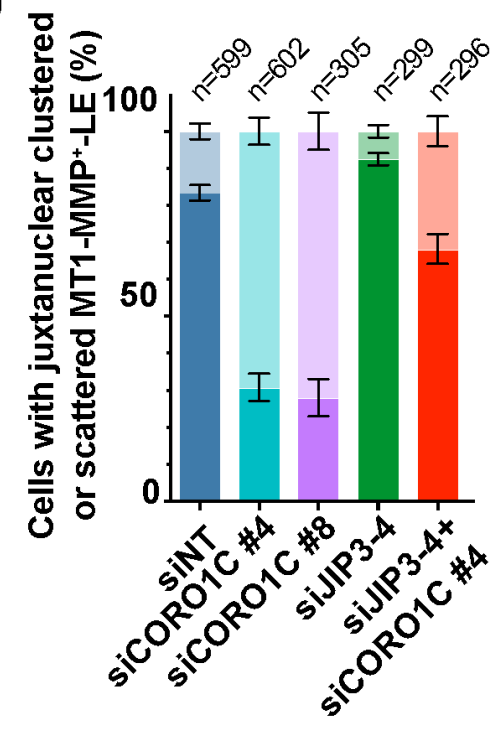

MT1-MMP Endosomes

Clustered

Scattered 


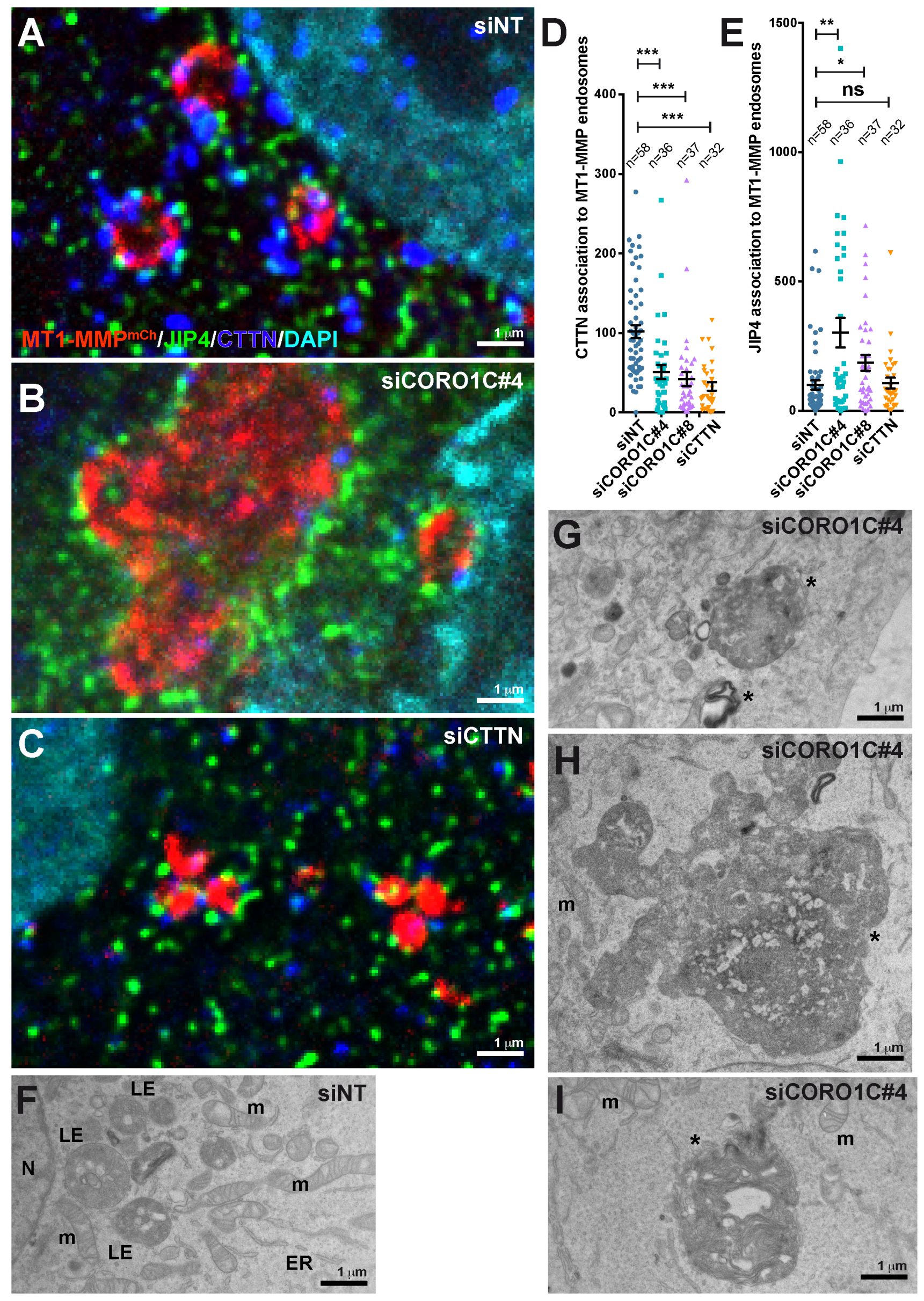

FIGURE 6 